\title{
SiO emission from low- and high-velocity shocks in Cygnus- $X$ massive dense clumps ${ }^{\star}$
}

\author{
A. Duarte-Cabral ${ }^{1,2,3}$, S. Bontemps ${ }^{2,3}$, F. Motte ${ }^{4}$, A. Gusdorf ${ }^{5}$, T. Csengeri ${ }^{6}$, N. Schneider ${ }^{2,3}$, and F. Louvet ${ }^{4}$ \\ ${ }^{1}$ School of Physics and Astronomy, University of Exeter, Stocker Road, Exeter EX4 4QL, UK \\ e-mail: adc@astro.ex.ac.uk \\ 2 Univ. Bordeaux, LAB, UMR 5804, 33270 Floirac, France \\ 3 CNRS, LAB, UMR 5804, 33270 Floirac, France \\ ${ }^{4}$ Laboratoire AIM, CEA/DSM-CNRS-Université Paris Diderot, IRFU/Service d'Astrophysique, C.E. Saclay, Orme de merisiers, \\ 91191 Gif-sur-Yvette, France \\ 5 LERMA, UMR 8112 du CNRS, Observatoire de Paris, École Normale Supérieure, 24 rue Lhomond, 75005 Paris, France \\ ${ }^{6}$ Max Planck Institute for Radioastronomy, Auf dem Hügel 69, 53121 Bonn, Germany
}

Received 19 February 2014 / Accepted 4 July 2014

\section{ABSTRACT}

\begin{abstract}
Context. The SiO molecule is formed through interstellar shocks and is often used as a tracer of high-velocity shocks from protostellar outflows. However, recent studies have suggested that low-velocity shocks in the interstellar medium can be responsible for a significant amount of $\mathrm{SiO}$ emission observed in star-forming regions.

Aims. We aim to investigate the existence of $\mathrm{SiO}$ emission that may or may not be associated with outflow shocks, within several massive dense clumps (MDCs), and explore how the SiO luminosities compare with the outflow properties estimated using CO.

Methods. We used observations of $\mathrm{SiO}(2-1)$ from the Plateau de Bure Interferomètre, towards a sample of six MDCs in Cygnus-X, and compared them to the $\mathrm{CO}$ high-velocity outflow emission, and to the velocity shears found in these regions.

Results. We find that most molecular outflows are detected in both $\mathrm{SiO}$ and $\mathrm{CO}$, although there are some cases of $\mathrm{CO}$ outflows with no $\mathrm{SiO}$ counterpart. The data also shows narrow line $\mathrm{SiO}$ emission $\left(\sigma_{v} \lesssim 1.5 \mathrm{~km} \mathrm{~s}^{-1}\right)$ which, in some cases, appears to be unrelated to outflows. The kinematics of this narrow emission often differs from those found by other high-density tracers such as $\mathrm{H}^{13} \mathrm{CO}^{+}$, and its extent varies from rather compact $(\sim 0.03 \mathrm{pc})$ to widespread $(\sim 0.2 \mathrm{pc})$. We find that the least centrally concentrated clumps with the least massive protostellar cores have the most widespread narrow $\mathrm{SiO}$ emission. The fraction of the total $\mathrm{SiO}$ luminosity that is not associated with outflows is highly variable in the different MDCs (from 10\% to 90\%); this might be a problem when extrapolating outflow properties from $\mathrm{SiO}$ luminosities without resolving individual outflows.

Conclusions. In line with previous evidence of $\mathrm{SiO}$ emission associated with low-velocity shocks, we propose an evolutionary picture to explain the existence and distribution of narrow $\mathrm{SiO}$ line profiles. In this scenario, the least centrally condensed MDCs are at an early stage where the $\mathrm{SiO}$ emission traces shocks from the large-scale collapse of material onto the MDC. This could be the case of CygX-N40, a MDC with a low-mass protostar at its centre, a weak outflow, and where $90 \%$ of the SiO emission is narrow and arises from the outskirts. As the MDC collapses, the $\mathrm{SiO}$ emission becomes more confined to the close surroundings of cores, tracing the post-shock material from the infalling MDC against the dense cores, such as in the small-scale converging flows of CygX-N3, N12, and N48. At later stages, when single massive protostars are formed, as for instance in CygX-N53 and N63, the SiO luminosity is largely dominated by powerful outflows, and the weaker narrow component shows perhaps the last remnants of the initial collapse.
\end{abstract}

Key words. stars: formation - stars: protostars - stars: massive - ISM: jets and outflows - ISM: kinematics and dynamics ISM: molecules

\section{Introduction}

The $\mathrm{SiO}$ molecule is the most abundantly observed siliconbearing molecular species in the interstellar medium (ISM), and it can be produced by the photo-evaporation of icy grain mantles (e.g. Turner 1991; Walmsley et al. 1999; Schilke et al. 2001; Godard \& Cernicharo 2013) or by the destruction of grain cores in shocks (e.g. Martin-Pintado et al. 1992; Schilke et al. 1997; Le Picard et al. 2001; Gusdorf et al. 2008a,b). The high-velocity shocks from protostellar outflows can easily produce $\mathrm{SiO}$, and therefore, observations of $\mathrm{SiO}$ emission are commonly used to trace such outflows (e.g. Beuther et al. 2002; Qiu et al. 2007; López-Sepulcre et al. 2011). Until recently, only fast molecular shocks were believed to be able to produce $\mathrm{SiO}$ by sputtering

\footnotetext{
* Data cubes in FITS format are only available at the CDS via anonymous ftp to cdsarc.u-strasbg.fr $(130.79 .128 .5)$ or via http://cdsarc.u-strasbg.fr/viz-bin/qcat?J/A+A/570/A1
}

of the grain cores (typically C-shocks with $v_{\mathrm{s}}>25 \mathrm{~km} \mathrm{~s}^{-1}$, but fast $J$-shocks can also form $\mathrm{SiO}$, see e.g. Guillet et al. 2009). Nevertheless, we do observe significant $\mathrm{SiO}$ emission at low velocities and with a small velocity dispersion. For instance, single-dish $\mathrm{SiO}$ pointed observations towards the Cygnus-X massive dense clumps (MDCs) by Motte et al. (2007) had shown evidence of two different gas components contributing to the $\mathrm{SiO}$ emission: a broad component tracing the shocked gas from protostellar outflows and a narrower one $\left(\sigma_{v} \lesssim 1.5 \mathrm{~km} \mathrm{~s}^{-1}\right.$, i.e. $F W H M \lesssim 3.5 \mathrm{~km} \mathrm{~s}^{-1}$ ) interpreted at the time as possibly arising from the hot cores. More recently, some individual studies have detected narrow and widespread $\mathrm{SiO}$ emission in molecular clouds, even in regions where no feedback from the forming protostars has yet kicked in (e.g. Jiménez-Serra et al. 2010; Nguyên-Lu'o'ng et al. 2013; Sanhueza et al. 2013; Kauffmann et al. 2013; Louvet et al., in prep.). The origin of $\mathrm{SiO}$ is in these cases enigmatic. 
Table 1. Observing parameters, beam sizes, and rms.

\begin{tabular}{|c|c|c|c|c|c|c|c|c|}
\hline \multirow{2}{*}{$\begin{array}{c}\text { Source } \\
\text { name }\end{array}$} & \multicolumn{2}{|c|}{ Phase centre (J2000) } & \multirow{2}{*}{$\begin{array}{c}\text { Synthesised beam } \\
{\left[{ }^{\prime \prime} \times{ }^{\prime \prime}\right]}\end{array}$} & \multirow{2}{*}{$\begin{array}{l}\mathrm{PA} \\
{\left[{ }^{\circ}\right]}\end{array}$} & \multirow{2}{*}{$\begin{array}{c}\mathrm{rms}^{a} \\
{[\mathrm{mJy} / \text { beam] }}\end{array}$} & \multicolumn{2}{|c|}{ Number of fragments ${ }^{b}$} & \multirow[b]{2}{*}{ Morphology } \\
\hline & $\mathrm{RA}\left(\mathrm{h} \mathrm{m} \mathrm{s}^{2}\right)$ & $\operatorname{Dec}\left({ }^{\circ}, \prime \prime\right)$ & & & & Total & High-mass & \\
\hline CygX-N3 & 203534.1 & 422005.0 & $3.68 \times 3.02$ & 63 & 12 & 4 & $2\left(>10 M_{\odot}\right)$ & Elongated \\
\hline CygX-N12 & 203657.4 & 421127.5 & $4.09 \times 3.51$ & 62 & 15 & 4 & $2\left(>15 M_{\odot}\right)$ & Elongated \\
\hline CygX-N40 & 203859.8 & 422342.0 & $3.64 \times 3.01$ & 64 & 12 & 1 & 0 & Diffuse \\
\hline CygX-N48 & 203901.5 & 422204.0 & $4.25 \times 3.35$ & 71 & 21 & 5 & $2\left(>10 M_{\odot}\right)$ & Clustered \\
\hline CygX-N53 & 203903.1 & 422550.0 & $4.23 \times 3.33$ & 70 & 21 & 7 & $2\left(>20 M_{\odot}\right)$ & Centrally condensed \\
\hline CygX-N63 & 204005.2 & 413212.0 & $4.12 \times 3.69$ & 46 & 15 & 3 & $1\left(>40 M_{\odot}\right)$ & Centrally condensed \\
\hline
\end{tabular}

Notes. ${ }^{(a)} 1 \sigma$ rms estimated in $0.27 \mathrm{~km} \mathrm{~s}^{-1}$ channels. ${ }^{(b)}$ From Bontemps et al. (2010).

Some models suggest that the formation of SiO from lowvelocity shocks could be linked to the presence of the element $\mathrm{Si}$ in the pre-shock medium. If there is some $\mathrm{Si}$ already in the gas phase, shocks with velocities as low as $\sim 5 \mathrm{~km} \mathrm{~s}^{-1}$ can produce SiO (e.g. Nguyên-Lu'o'ng et al. 2013). On the other hand, despite only considering silicates in grain cores, Caselli et al. (1997) demonstrated that grain-grain collisions dominate over sputtering for evaporation of the ices on the grain mantles for low-velocity shocks $\left(10-15 \mathrm{~km} \mathrm{~s}^{-1}\right)$. In this context, if a fraction of $\mathrm{Si}$ is in the grain ice mantles, then shock velocities of $\sim 10 \mathrm{~km} \mathrm{~s}^{-1}$ can desorb $\mathrm{Si}$ into the gas phase and effectively increase the abundances of $\mathrm{SiO}$ to match observations (e.g. Gusdorf et al. 2008b; Jiménez-Serra et al. 2008). If the Si is solely in the grain cores, then shock velocities above $25 \mathrm{~km} \mathrm{~s}^{-1}$ are needed to form $\mathrm{SiO}$ (e.g. Schilke et al. 1997; Gusdorf et al. 2008a). However, none of these models include grain-grain interactions. To our knowledge, the most sophisticated account of these interactions has been given by the studies of Guillet et al. (2007, 2009, 2011) and Anderl et al. (2013). When included in shock models, these interactions (namely vaporisation, coagulation, and shattering) do significantly affect the structure of C-type shock waves propagating in high pre-shock density conditions $\left(n_{\mathrm{H}}>10^{5} \mathrm{~cm}^{-3}\right)$. Because they result in the production of numerous small grains, the modelled shock layers are both thinner and hotter. Ultimately, even if collisional vaporisation becomes more efficient than sputtering at destroying grains, especially at slightly lower shock velocities $\left(20-25 \mathrm{~km} \mathrm{~s}^{-1}\right)$, the overall result of these improved models is less bright and narrower $\mathrm{SiO}$ line profiles that do not match the observed intensities, unless a fraction of silicon-bearing material is placed in the grain mantles, similar to what Gusdorf et al. (2008b) had done.

It is vital to further investigate the origin of the measured $\mathrm{SiO}$ components, to be able to disentangle $\mathrm{SiO}$ outflows from other possible forms of shocks. However, most existing surveys observing $\mathrm{SiO}$ emission towards star-forming regions do not possess enough angular resolution or sensitivity to determine the exact origin of the $\mathrm{SiO}$ emission, and it becomes impossible to disentangle any emission that may not arise from outflows. On the one hand, ignoring other sources of shocks for the origin of $\mathrm{SiO}$ could introduce significant uncertainties on any outflow properties derived from $\mathrm{SiO}$ luminosities alone. On the other hand, pinpointing the exact origin of $\mathrm{SiO}$ emission can help to understand the physics and mechanisms for accretion at core-scales.

In this article, we present $\mathrm{SiO}(2-1)$ interferometric observations towards a sample of IR-quiet MDCs in Cygnus-X, located at a distance of $1.4 \mathrm{kpc}$ (Rygl et al. 2012), which are known to have both broad and narrow SiO emission (Motte et al. 2007), and for which we have detailed information about the protostellar content and the existing outflows (Duarte-Cabral et al. 2013).
We address the consequences of not resolving the $\mathrm{SiO}$ emission for inferring outflow properties, and we investigate the different possibilities for the origin of the observed $\mathrm{SiO}$ emission. Details on the source sample and observations are provided in Sect. 2. We describe our results in Sect. 3, which include a comparison of the spectral profiles of $\mathrm{SiO}$ with those obtained with singledish observations, and a comparison of the $\mathrm{SiO}$ and $\mathrm{CO}$ outflow emission. We analyse the different trends observed with $\mathrm{SiO}$ and the emission morphologies in Sect. 4, and present our concluding remarks in Sect. 5.

\section{Source sample}

\subsection{Cygnus- $X$ massive dense clumps}

We have studied the six IR-quiet MDCs in Cygnus-X, originally identified by Motte et al. (2007) and more recently observed with the PdBI in the $1 \mathrm{~mm}$ and $3 \mathrm{~mm}$ continuum emission (Bontemps et al. 2010) and ${ }^{12} \mathrm{CO}(2-1)$ outflow emission (Duarte-Cabral et al. 2013). Table 1 lists the name and properties of each MDC, including the number of fragments and morphology.

These MDCs are found in a range of different environments, and each field, with the exception of CygX-N40, is subfragmented into several cores with envelope masses larger than $\sim 10 M_{\odot}$ (as estimated from the $1 \mathrm{~mm}$ emission by Bontemps et al. 2010; Duarte-Cabral et al. 2013). The CygX-N3 and N12 MDCs are situated to the west of the DR21 ridge and they both show elongated morphologies, consistent with being shaped by the winds from the nearby OB clusters (to the N and NW). Both are fragmented into several compact cores, with two high-mass cores per field (see Table 1). CygX-N40, N48, and N53 are located along the massive DR21 ridge, a highly dynamical star formation site (Schneider et al. 2010; Hennemann et al. 2012). The interferometric continuum data of CygX-N40 (from Bontemps et al. 2010) showed that this core is not centrally condensed, with its emission being extended and filtered out by the PdBI. Bontemps et al. (2010) had detected a single low-mass fragment in this region, with $M_{\text {env }}<2 M_{\odot}$. CygX-N48 has a clustered star formation, not very centrally condensed, but with two massive compact sources, while CygX-N53 is centrally condensed and fragmented into two compact massive cores (with more than $20 M_{\odot}$ each). Finally, CygX-N63 is a relatively isolated dense core to the south of DR21. It does not show signs of fragmentation and has nearly $50 M_{\odot}$ within its inner $\sim 2500 \mathrm{AU}$, making it the most massive core of the sample.

In Duarte-Cabral et al. (2013), we confirmed the nature of these millimetre cores as high-mass Class 0-like protostars, based on their masses, luminosities, and outflow power/accretion rates. We identified the individual outflows powered by each massive protostar, and estimated their properties, concluding 
Table 2. Systemic velocities and $\mathrm{SiO}$ line properties.

\begin{tabular}{|c|c|c|c|c|c|c|c|c|c|c|c|}
\hline \multirow[b]{2}{*}{ Source } & \multirow{2}{*}{$\begin{array}{c}\mathrm{N}_{2} \mathrm{H}^{+} \\
v_{0} \\
\left(\mathrm{~km} \mathrm{~s}^{-1}\right) \\
\end{array}$} & \multicolumn{3}{|c|}{ Narrow component } & \multicolumn{3}{|c|}{ Broad component } & \multicolumn{2}{|c|}{ Total flux (in $\left.30^{\prime \prime}\right)^{*}$} & \multicolumn{2}{|c|}{ High- $v$ range } \\
\hline & & $\begin{array}{c}I_{\text {peak }}^{n} \\
(\mathrm{Jy})\end{array}$ & $\begin{array}{c}v_{\text {peak }}^{n} \\
\left(\mathrm{~km} \mathrm{~s}^{-1}\right)\end{array}$ & $\begin{array}{c}\sigma_{v}^{n} \\
\left(\mathrm{~km} \mathrm{~s}^{-1}\right)\end{array}$ & $\begin{array}{c}I_{\text {peak }}^{b} \\
(\mathrm{Jy})\end{array}$ & $\begin{array}{c}v_{\text {peak }}^{b} \\
\left(\mathrm{~km} \mathrm{~s}^{-1}\right)\end{array}$ & $\begin{array}{c}\sigma_{v}^{b} \\
\left(\mathrm{~km} \mathrm{~s}^{-1}\right)\end{array}$ & $\begin{array}{l}\text { PdBI } \\
\text { (Jy) }\end{array}$ & $\begin{array}{l}\text { SD } \\
(\mathrm{Jy})\end{array}$ & $\begin{array}{l}\text { Blue } \\
\left(\mathrm{km} \mathrm{s}^{-1}\right)\end{array}$ & $\begin{array}{c}\text { Red } \\
\left(\mathrm{km} \mathrm{s}^{-1}\right)\end{array}$ \\
\hline CygX-N3 & 14.9 & 1.4 & $15.5 \pm 0.2$ & $1.6 \pm 0.3$ & 0.7 & $17.0 \pm 0.8$ & $3.8 \pm 0.6$ & 13.6 & 14.4 & {$[-20.0 ; 11.9]$} & {$[17.9 ; 40.0]$} \\
\hline CygX-N12 & 15.2 & 2.5 & $12.9 \pm 0.1$ & $1.0 \pm 0.1$ & 1.9 & $12.2 \pm 0.2$ & $4.0 \pm 0.2$ & $9.1(30.1)^{* *}$ & 25.1 & {$[-10.0 ; 12.2]$} & {$[18.2 ; 25.0]$} \\
\hline CygX-N40 & -3.5 & 1.9 & $-3.1 \pm 0.1$ & $1.2 \pm 0.1$ & 0.5 & $-4.5 \pm 0.8$ & $7.2 \pm 0.9$ & $3.9(19.4)^{* *}$ & 12.6 & {$[-35.0 ;-6.5]$} & {$[-0.5 ; 30.0]$} \\
\hline CygX-N48 & -3.5 & 2.7 & $-4.2 \pm 0.1$ & $2.0 \pm 0.1$ & 0.3 & $-10.5 \pm 2.0$ & $14.6 \pm 2.1$ & 22.9 & 28.7 & {$[-35.0 ;-6.5]$} & {$[-0.5 ; 27.0]$} \\
\hline CygX-N53 & -4.4 & - & - & - & - & - & - & 34.4 & 48.5 & {$[-35.0 ;-7.4]$} & {$[-1.4 ; 26.0]$} \\
\hline CygX-N63 & -4.5 & 0.8 & $-6.0 \pm 0.3$ & $2.5 \pm 0.3$ & 0.4 & $-5.3 \pm 1.4$ & $14.9 \pm 1.7$ & 20.7 & 15.2 & {$[-50.0 ;-7.5]$} & {$[-1.5 ; 50.0]$} \\
\hline
\end{tabular}

Notes. ${ }^{(*)}$ The total flux was estimated using the integrated intensities over the entire velocity range (as plotted in Fig. 1), and they are the same for the single-dish (SD) and PdBI data. ${ }^{(*)}$ The values inside brackets show the PdBI flux retrieved from a $50^{\prime \prime} \times 50^{\prime \prime}$ region instead of $30^{\prime \prime} \times 30^{\prime \prime}$.

that the opening angles and morphologies are similar to those of low-mass objects, and they are 1-2 orders of magnitude more powerful, following a linear trend with the envelope mass.

\subsection{Observations}

The six MDCs were observed in 2004 with the PdBI in the $1.3 \mathrm{~mm}$ and $3.5 \mathrm{~mm}$ continuum emission and in four spectral units covering the ${ }^{12} \mathrm{CO}(2-1), \mathrm{SiO}(2-1), \mathrm{H}^{13} \mathrm{CO}^{+}(1-0)$, and $\mathrm{H}^{13} \mathrm{CN}(1-0)$ lines. The observations in the continuum and in the $\mathrm{H}^{13} \mathrm{CO}^{+}(1-0)$ and $\mathrm{H}^{13} \mathrm{CN}(1-0)$ lines are reported in Bontemps et al. (2010) and Csengeri et al. (2011a). The ${ }^{12} \mathrm{CO}(2-1)$ observations (shown in Duarte-Cabral et al. 2013) have been shortspaced using IRAM $30 \mathrm{~m}$ data, and will be used here to aid the identification of individual outflows. This article presents the $\mathrm{SiO}(2-1)$ observations, at $86.85 \mathrm{GHz}$, with an angular resolution of $\sim 3^{\prime \prime}$ (i.e. $\sim 0.02 \mathrm{pc}$ at $1.4 \mathrm{kpc}$ distance), tracing interstellar shocks at core-scales (typical protostellar outflows being $\sim 0.1-0.4$ pc in length).

The observations were performed in track-sharing mode with two targets per track for the following pairs: CygX-N48/N53, CygX-N3/N40, and CygX-N12/N63. The $\mathrm{D}$ configuration track observations were performed between June and October 2004 (five antennas with baselines between $24 \mathrm{~m}$ and $82 \mathrm{~m}$ ). The $\mathrm{C}$ configuration tracks were obtained in November and December 2004 (six antennas in 6Cp with baselines from $48 \mathrm{~m}$ to $229 \mathrm{~m}$ ). The bright nearby quasar $2013+370$ was used as a phase calibrator, and the evolved star MWC349 as a flux calibrator.

The maps were cleaned using the natural weighting, and the resulting synthesised beam and root mean square (rms) in the continuum are summarised in Table 1, together with the field names and centres of phase. The pixel size is of $0.62^{\prime \prime}$. The cleaning components were searched across the whole area of the primary beam. No support for cleaning was used to avoid introducing any bias into the resulting emission maps.

\section{Results}

\subsection{Profile of the SiO emission}

Motte et al. (2007) presented single-dish pointed observations of $\mathrm{SiO}(2-1)$ emission with the IRAM $30 \mathrm{~m}$ telescope (beam size of $\sim 29^{\prime \prime}$ ) towards the sample of IR-quiet cores we study here. Figure 1 shows the $\mathrm{SiO}$ average profiles from our PdBI observations as dark grey shadowed spectra (averaged within the central $30^{\prime \prime} \times 30^{\prime \prime}$ ) overlaid with the dotted blue spectra from the single-dish observations.
There is a good correspondence between the two profiles for 3 out of 6 sources (CygX-N3, N48 and N63). For these three MDCs, the PdBI recovers most of the flux detected with the single-dish $(95 \%, 80 \%$, and $>100 \%$ for CygX-N3, N48, and N63, respectively; see Table 2). The fact that we recover slightly more emission with the PdBI with respect to the IRAM $30 \mathrm{~m}$ telescope in N63 can be explained by the uncertainties on the calibration of the IRAM $30 \mathrm{~m}$ data $(\sim 10 \%)$, by some loss of power towards the edges of the single-dish primary beam, and by the fact that the area we used to extract the average PdBI profiles is a rectangular area of $30^{\prime \prime}$ width, instead of a circular beam shape. In CygX-N53, even though the shape of the profile is similar, we miss some of the systemic velocity emission with the PdBI. Nevertheless, we still recover $\sim 70 \%$ of the single-dish flux with the PdBI. This indicates that most of the $\mathrm{SiO}$ emission is compact on these four sources, and not filtered out with the PdBI.

The two most striking exceptions are CygX-N12 and N40, where a significant fraction of the single-dish flux $(\sim 65-70 \%)$ is missing with the PdBI, when only the central $30^{\prime \prime}$ emission is taken, either because some flux is filtered out in the interferometric observations or because the single-dish observations suffer from contamination from strong emission sitting in the immediacies of the single-dish primary beam. In order to discriminate between these possibilities, we have integrated the PdBI spectra for these two regions considering a larger area of $50^{\prime \prime} \times 50^{\prime \prime}$ (light grey shadowed spectra in the panels of these two sources). Doing so for CygX-N12 recovers not only the flux of the singledish observations, but also the line shape. We conclude that this is due to the contamination from the region east of the singledish primary beam, with strong outflow emission (see central panels of Fig. 2). Doing this for CygX-N40, despite a considerable improvement, still does not recover the peak flux detected with the single-dish at systemic velocities, suggesting that there is a considerable amount of extended $\mathrm{SiO}$ emission at systemic velocities which is filtered by the PdBI (with a need for shortspacings). On the other hand, it recovers some line-wings that were not seen with the single-dish.

Based on the single-dish SiO emission, Motte et al. (2007) discussed the existence of two components of $\mathrm{SiO}$ emission. Our observations confirm that the average spectra of most sources can be accurately represented by the emission of two Gaussian components (Fig. 1), with the exception of CygX-N53 which shows a clear asymmetric profile, with broad redshifted emission (reaching a terminal velocity of $\sim 30 \mathrm{~km} \mathrm{~s}^{-1}$ ). In Table 2 we show the systemic velocities, $v_{0}$, of the different regions as measured by Bontemps et al. (2010) using $\mathrm{N}_{2} \mathrm{H}^{+}$pointed observations, also performed with the IRAM $30 \mathrm{~m}$ telescope (and 

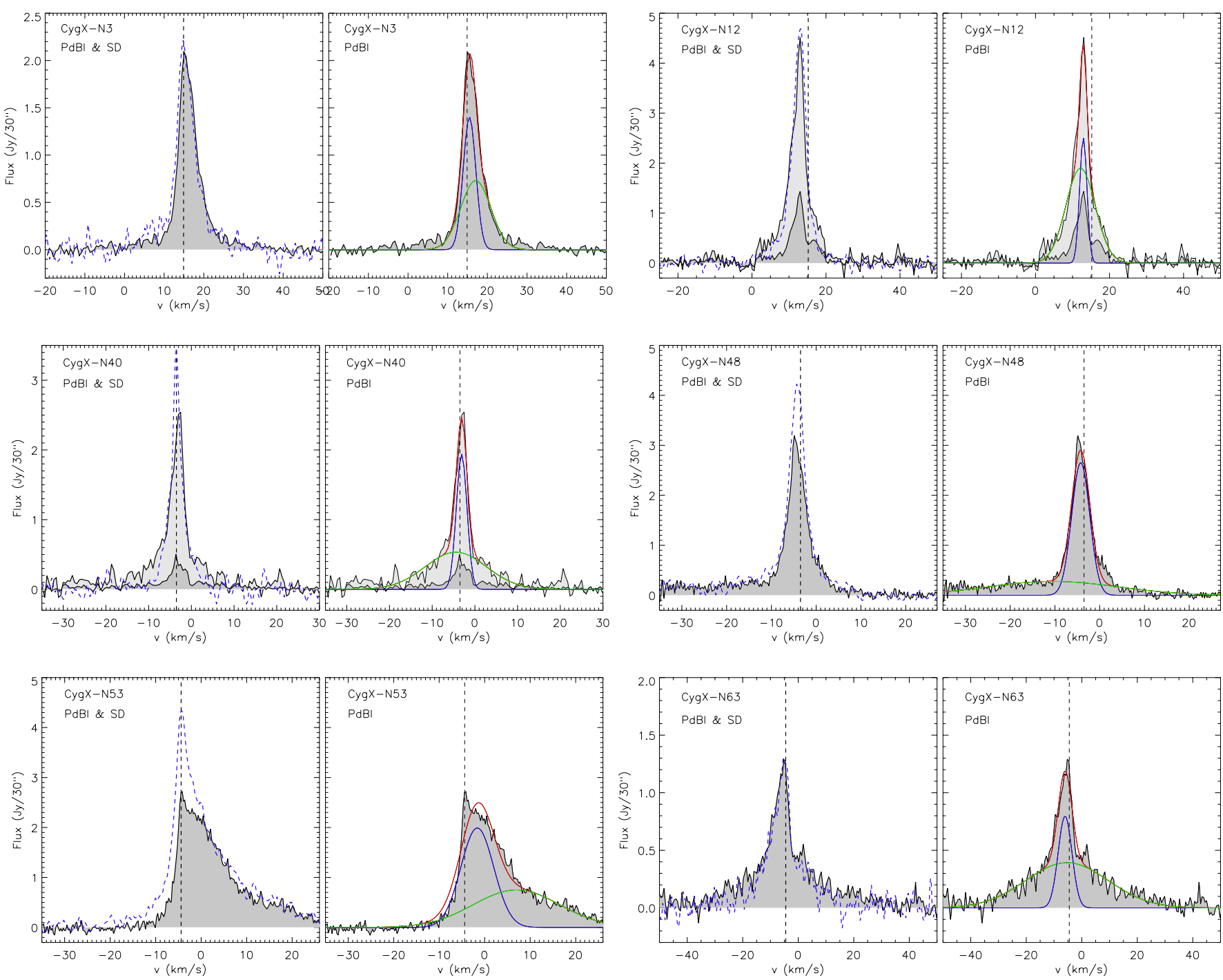

Fig. 1. Average spectra for the six MDCs of our sample (labelled on the top-left corners). The left side of each subfigure shows the comparison of the single-dish (SD) IRAM $30 \mathrm{~m}$ pointed observations (Motte et al. 2007) in blue dashed lines, and the spectra of our PdBI SiO observations integrated over the central $30^{\prime \prime} \times 30^{\prime \prime}$ (equivalent to the IRAM $30 \mathrm{~m}$ beam) are shadowed in grey. The vertical black dashed lines show the systemic velocity of each core, as measured with $\mathrm{N}_{2} \mathrm{H}^{+}$by Bontemps et al. (2010). The right side shows the same PdBI SiO emission as the left, overplotted with a two-component Gaussian fit to illustrate the existence of narrow (in blue) and broad (in green) components of emission (whose sum is shown in red). For CygX-N12 and N40, we show a shadowed spectrum (in light grey) which corresponds to the PdBI emission integrated over an area of $50^{\prime \prime} \times 50^{\prime \prime}$ (to assess a possible contamination from strong $\mathrm{SiO}$ emission outside the SD primary beam).

therefore within an equivalent beam size). The uncertainties on these velocities are of $0.1 \mathrm{~km} \mathrm{~s}^{-1}$. These are shown as dashed vertical lines in Fig. 1. For the MDCs where two Gaussian components fit well the observed average spectra, we present, in Table 2 , the peak intensity, $I_{\text {peak }}$; the peak velocity, $v_{\text {peak }}$; and the velocity dispersion, $\sigma_{v}$, for both the narrow and the broad components. The peak of the narrow component of the $\mathrm{SiO}$ emission is only slightly different from the systemic velocities (less than $1 \mathrm{~km} \mathrm{~s}^{-1}$ difference), which is not significant considering the linewidths of the emission and the uncertainties on the peak velocity. This is with the exception of N12, where there is an important shift between the two (of $2 \mathrm{~km} \mathrm{~s}^{-1}$ ). We will discuss possible explanations for this shift later in the article (Sect. 4.2.1).

While the broad emission whose average width at the base reaches $\gtrsim 30 \mathrm{~km} \mathrm{~s}^{-1}$ suggests unequivocally outflow shocks, Motte et al. (2007) considered the possibility of the narrower component originating from shocks inside a hot core and/or lower velocity outflows. The only way to distinguish between a high-velocity or low-velocity shock origin is by carefully inspecting the spectra along the maps, and investigate the spatial distribution of the systemic-velocity and broad emissions (see Sects. 3.2 and 3.3). The spectra throughout the maps vary significantly from the average spectra, and examples of the different line profiles found in each MDC can be found in Appendix A, Fig. A.2.

\subsection{Distribution of high-velocity $\mathrm{SiO}$ versus $\mathrm{CO}$}

To investigate how effectively $\mathrm{SiO}$ is tracing protostellar outflows, we compare the spatial distribution of the high-velocity $\mathrm{SiO}$ emission with that of CO (Duarte-Cabral et al. 2013). Table 2 shows the velocity ranges used to estimate the $\mathrm{SiO}$ integrated intensities and luminosities at high-velocities (high- $v$ range), which is in essence the emission offset from the systemic velocity of the cloud by more than $\pm 3 \mathrm{~km} \mathrm{~s}^{-1}$ (corresponding to roughly $3 \sigma_{v}$ from the fits to the $\mathrm{N}_{2} \mathrm{H}^{+}(1-0)$ spectra by 

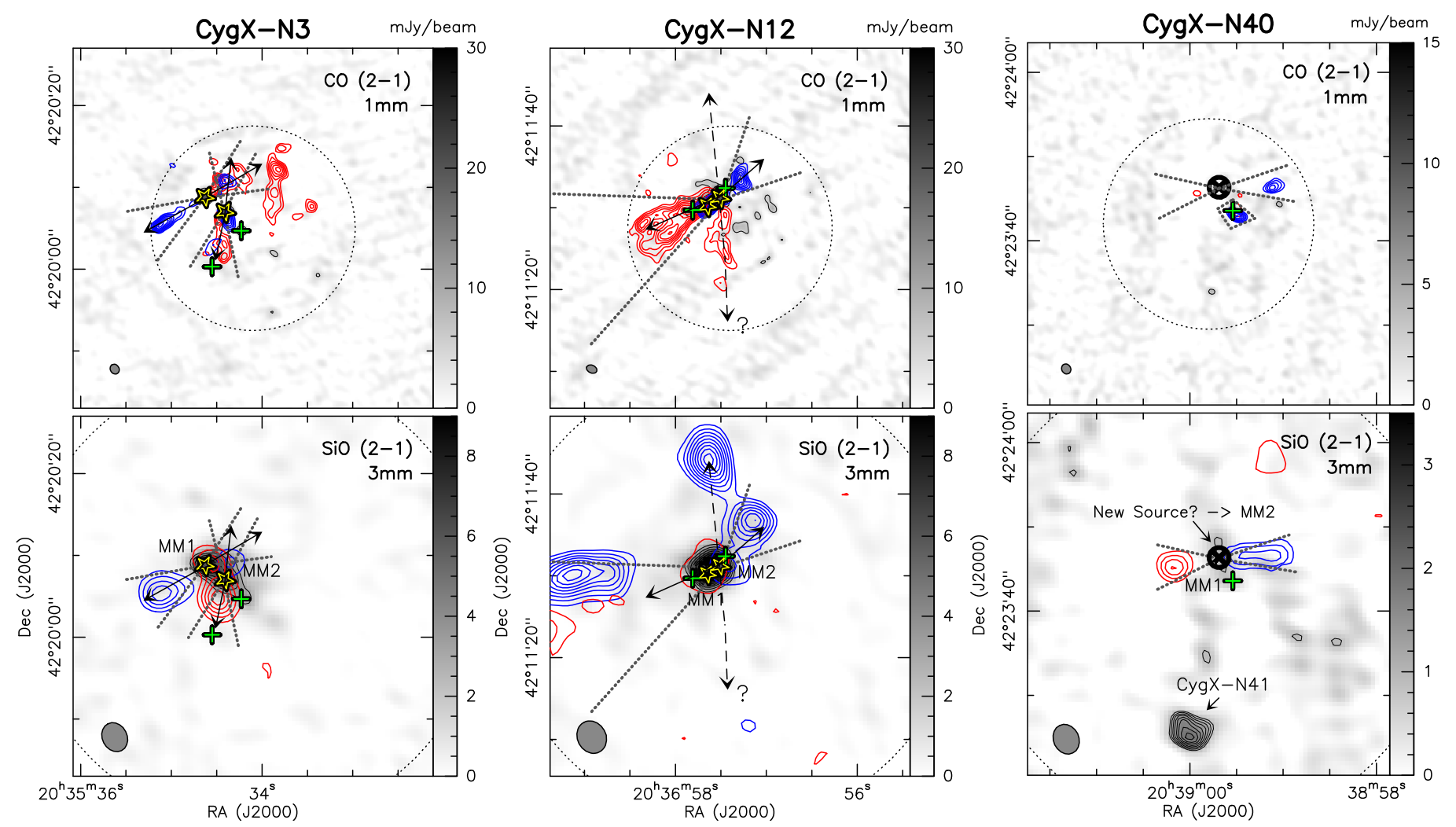

Fig. 2. Comparison of the high-velocity $\mathrm{CO}$ and SiO emission in CygX-N3 (left), N12 (centre), and N40 (right). The top panels show the highvelocity CO emission in contours (with the velocity ranges as in Duarte-Cabral et al. 2013) over the $1 \mathrm{~mm}$ continuum emission in greyscale. The primary beam of the $\mathrm{CO}$ and $1 \mathrm{~mm}$ observations is shown as a dashed circle, and the synthetic beam is in the lower-left corner. Contours are stepped by $0.25 \mathrm{Jy} \mathrm{beam}^{-1} \mathrm{~km} \mathrm{~s}^{-1}$, starting at 2.0 (2.75) Jy beam ${ }^{-1} \mathrm{~km} \mathrm{~s}^{-1}$ for N3 blue (red), $1.5 \mathrm{Jy} \mathrm{beam}^{-1} \mathrm{~km} \mathrm{~s}^{-1}$ for N12, and 1.75 (1.25) Jy beam ${ }^{-1} \mathrm{~km} \mathrm{~s}^{-1}$ for $\mathrm{N} 40$ blue (red). The lower panels show the high-velocity $\mathrm{SiO}$ emission in contours (with velocity ranges as in Table 2) over the $3 \mathrm{~mm}$ continuum emission in greyscale for the same sources. The primary beams are shown as dashed circles and the synthetic beams are plotted in the lower-left corners. Contours are stepped by $0.1 \mathrm{Jy} \mathrm{beam}^{-1} \mathrm{~km} \mathrm{~s}^{-1}$, starting at $0.15 \mathrm{Jy} \mathrm{beam}^{-1} \mathrm{~km} \mathrm{~s}^{-1}$ for N3, $0.2(0.05) \mathrm{Jy} \mathrm{beam}^{-1} \mathrm{~km} \mathrm{~s}^{-1}$ for N12 blue (red), and $0.2 \mathrm{Jy} \mathrm{beam}^{-1} \mathrm{~km} \mathrm{~s}^{-1}$ for $\mathrm{N} 40$. The most massive protostars are marked with yellow stars (and labelled in the lower panels), and the least massive fragments from Bontemps et al. (2010) are shown as green crosses. The arrows are the directions of the outflows inferred from Duarte-Cabral et al. (2013), and the dashed lines show the respective outflow cones.

Bontemps et al. 2010). Figures 2 and 3 show the high-velocity $\mathrm{CO}$ and $\mathrm{SiO}$ emission in the top and bottom panels, respectively. In these figures we also show the outflow cones (dotted lines) and directions (arrows). Although with a different resolution $\left(\sim 1^{\prime \prime}\right)$ and field of view (see dashed circles in Figs. 2 and 3), the $\mathrm{CO}$ is used as a reference to identify the different outflow lobes and respective driving sources.

From a fast inspection of these figures we can see a number of similarities in the distribution of the high-velocity $\mathrm{SiO}$ and high-velocity $\mathrm{CO}$ outflow emission. In particular, for CygX-N3 (left column of Fig. 2) and CygX-N63 (right column of Fig. 3), the correspondence is quite clear both spatially and in terms of relative intensity of the blue- and redshifted outflow lobes.

In other regions, the correspondence in terms of spatial extent and intensities is poorer. This is the case, for instance, of CygX-N53 (central column of Fig. 3) where SiO is almost only redshifted while $\mathrm{CO}$ is roughly symmetric. This is puzzling. Perhaps this is due to the powerful red lobe encountering more material which is efficiently shocked to form and entrain SiO. On the other hand, the blueshifted $\mathrm{SiO}$ traces only vaguely the blueshifted emission of CO. In addition, the redshifted emission seen towards the west could be due to an additional outflow, since there is a blueshifted counterpart in the same direction towards the east, at the edge of the primary beam. However, although it may be that the corresponding $\mathrm{CO}$ emission is outside our field of view, the absence of such outflow in $\mathrm{CO}$ makes it hard to understand its origin. It could be that it arises from CygX-N53 MM2, but this is not the outflow we consider for this source. Duarte-Cabral et al. (2013) suggest a tentative detection of a very compact outflow using $\mathrm{CO}$ emission for which we estimate the respective $\mathrm{SiO}$ luminosities here (and hence these are only to be taken as an upper limit). For CygX-N48 (left column of Fig. 3), there is also some discrepancy between the intensities of the two molecules, namely on the outflow powered by MM2. In this region, only $25 \%$ of the high-velocity SiO luminosity arises from the outflows of MM1 and MM2, and most high-velocity outflow emission in this field is due to the outflow from IS-1 (IS-1 stands for Infrared Source 1, and corresponds to a resolved source detected in the IRAC bands at 3 and $4 \mu \mathrm{m}$, and unresolved at $8 \mu \mathrm{m}$ and onwards, at RA $20^{\mathrm{h}} 39^{\mathrm{m}} 02.927^{\mathrm{s}}$ and Dec $42^{\circ} 22^{\prime} 07.32^{\prime \prime}$; using Herschel, Hennemann et al. (in prep.) define this source as a more evolved object). This demonstrates the need to identify individual outflows to perform good evaluations of individual outflow power (unresolved observations of this region would have assigned the emission from IS-1 outflow to the stronger millimetre peak, MM1).

Finally, other regions have high-velocity $\mathrm{SiO}$ emission at slightly different positions to where we had previously detected the CO outflows. For instance, in CygX-N12 (central column of Fig. 2), because of the larger field of view of the 

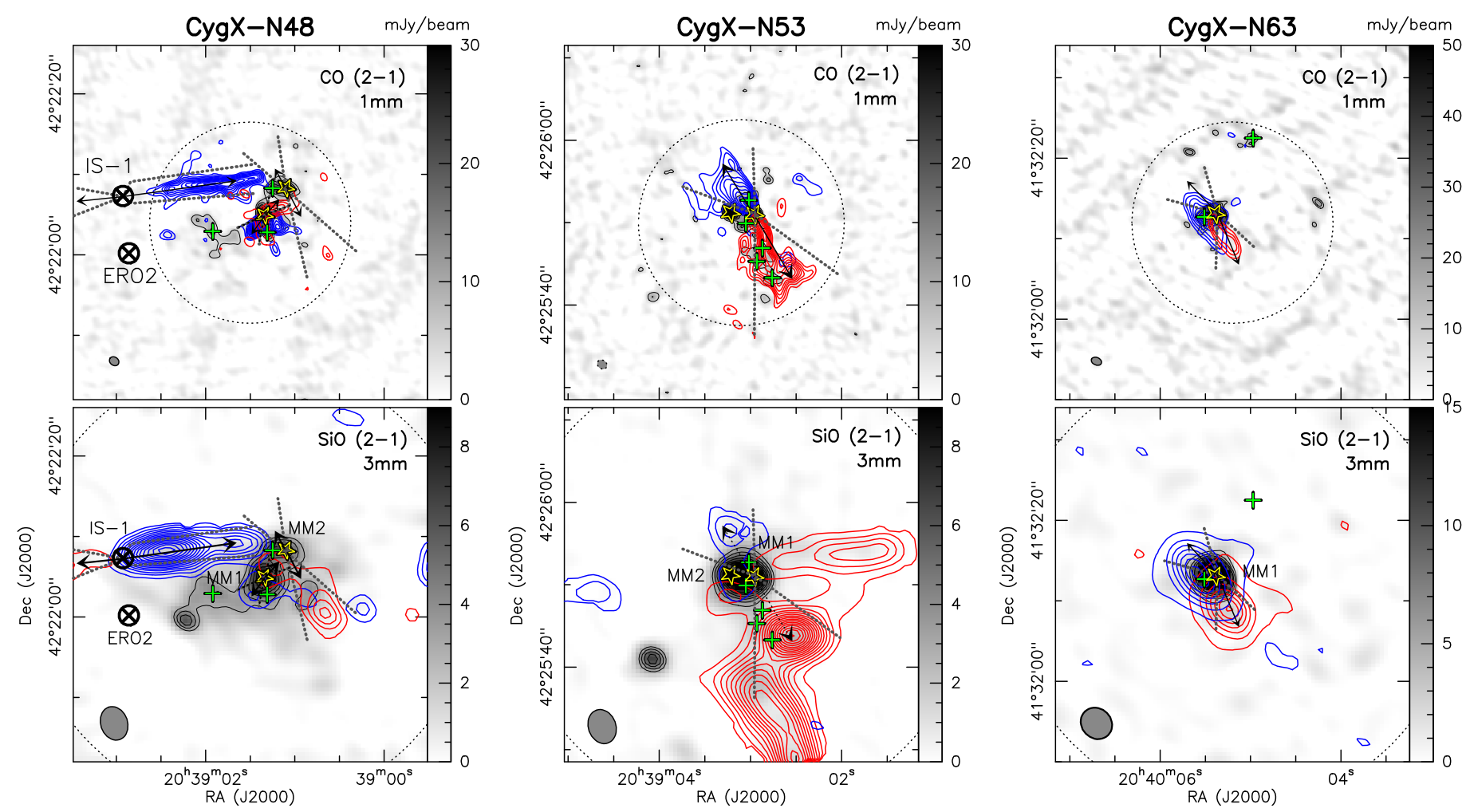

Fig. 3. Same as Fig. 2 for CygX-N48 (left), N53 (centre), and N63 (right). In N48 we mark with circled crosses two Spitzer sources: ERO2 (Marston et al. 2004) and IS-1, which is a resolved source detected in the IRAC bands at 3 and $4 \mu \mathrm{m}$, and unresolved at $8 \mu \mathrm{m}$ and onwards. IS- 1 is likely responsible for the outflow directed east-west. Contour key for CO emission, top panels: N48 (left) contour steps of $0.5 \mathrm{Jy} \mathrm{beam}^{-1} \mathrm{~km} \mathrm{~s}^{-1}$ starting at 4 (3) Jy beam ${ }^{-1} \mathrm{~km} \mathrm{~s}^{-1}$ for blue (red); N53 (centre) contour steps of $1.5 \mathrm{Jy} \mathrm{beam}^{-1} \mathrm{~km} \mathrm{~s}^{-1}$ starting at $8.5 \mathrm{Jy} \mathrm{beam}^{-1} \mathrm{~km} \mathrm{~s}^{-1}$; N63 (right) contour steps of $1.5 \mathrm{Jy} \mathrm{beam}^{-1} \mathrm{~km} \mathrm{~s}^{-1}$ starting at $5.0 \mathrm{Jy} \mathrm{beam}^{-1} \mathrm{~km} \mathrm{~s}^{-1}$. Contours of $\mathrm{SiO}$ emission, lower panels: N48 (left) contour steps of $0.1 \mathrm{Jy}_{\text {beam }}^{-1} \mathrm{~km} \mathrm{~s}^{-1}$, starting at $0.2(0.15) \mathrm{Jy} \mathrm{beam}^{-1} \mathrm{~km} \mathrm{~s}^{-1}$ for blue (red); N53 (centre) contour steps of $0.15(0.25) \mathrm{Jy} \mathrm{beam}^{-1} \mathrm{~km} \mathrm{~s}^{-1}$, starting at 0.2 (0.15) Jy beam ${ }^{-1} \mathrm{~km} \mathrm{~s}^{-1}$ for blue (red); N63 (right) contour steps of $0.5 \mathrm{Jy} \mathrm{beam}{ }^{-1} \mathrm{~km} \mathrm{~s}^{-1}$, starting at $0.2 \mathrm{Jy} \mathrm{beam}^{-1} \mathrm{~km} \mathrm{~s}^{-1}$, for both blue- and redshifted emission.

$\mathrm{SiO}$ observations, any outflow at the edge of the $\mathrm{SiO}$ maps was not covered by $\mathrm{CO}$. Close to the sources, the high-velocity emission from $\mathrm{CO}$ and $\mathrm{SiO}$ is similar (redshifted), and the larger extent of the $\mathrm{SiO}$ emission to the east could indicate that the red $\mathrm{CO}$ outflow becomes blue farther away, which suggests that this is an outflow cone close to the plane of the sky. Although it could be that the $\mathrm{SiO}$ blue lobe is simply part of another outflow (and unrelated with the CO red outflow lobe), its small velocity range $\left(\sim 10 \mathrm{~km} \mathrm{~s}^{-1}\right)$ and large spatial extent $\left(\sim 20^{\prime \prime}\right.$, i.e. $\left.\sim 0.13 \mathrm{pc}\right)$ support the hypothesis of an outflow close to the plane of the sky. There is also some blue high-velocity $\mathrm{SiO}$ emission to the north which could be due to a second outflow not detected in $\mathrm{CO}$ (and which would follow the opposite direction of the southdirected CO lobe, marked with a dashed line). Using CO only, in Duarte-Cabral et al. (2013), we had interpreted this region as having two overlapping outflows, both E-W directed. With $\mathrm{SiO}$, this picture could perhaps be revised with the existence of a N-S outflow, but because the emission does not trace back clearly to any of the two sources, we have refrained from doing so. Instead, and for the remainder of the paper, we will take into account only the outflow directed E-W. In CygX-N40 (right column of Fig. 2), the outflow emission we had detected with $\mathrm{CO}$ in Duarte-Cabral et al. (2013) close to MM1 has no SiO counterpart (perhaps it is too weak to be detected within out noise levels), while the two weak high-velocity lobes to the northeast and north-west of MM1 are detected with both molecules. This, along with the existing weak $3 \mathrm{~mm}$ peak, lead us to think that there is a second low-mass source $\left(M_{\text {env }} \lesssim 1 M_{\odot}\right)$, to the north of MM1, and which could be responsible for the additional
E-W CO and SiO outflow. We will refer to this source as CygXN40 MM2, whose position and estimate of the $F_{\mathrm{CO}}$ (as done for the other sources in Duarte-Cabral et al. 2013) is shown in Table. 3. In this region, only $10 \%$ of the total PdBI SiO luminosity is close to the central sources (MM2 in particular), $90 \%$ of which is high-velocity emission.

\subsection{Distribution of the narrow $\mathrm{SiO}$ emission}

We have investigated the distribution of the $\mathrm{SiO}$ emission close to the systemic velocities of the cloud, and whose main contribution is adequately fitted by a narrow Gaussian (see spectra in Fig. 1). Since sometimes the narrow $\mathrm{SiO}$ emission is along the same line of sight as some high-velocity gas, separating the contribution of the outflows from the emission at systemic velocities becomes critical. In an exercise to try and remove this contribution, we have created datacubes of the narrow emission of each field, by subtracting the contribution from a broad outflow profile whenever there was significant high-velocity emission. To do so, we selected all the pixels where the integrated high-velocity $\mathrm{SiO}$ emission was above the rms noise level, and fitted a double Gaussian profile to the spectrum at each pixel. The fitted broad Gaussian component was then subtracted from the original spectra. By doing so, we do not make an a priori assumption on the profile of the narrow component. Instead, we remove the broad contribution and analyse what is left from this process (see Fig. A.1 in Appendix A for an example spectrum before and after the subtraction of the broad component). Figures 4 and 5 show the spatial distribution of the moment maps 

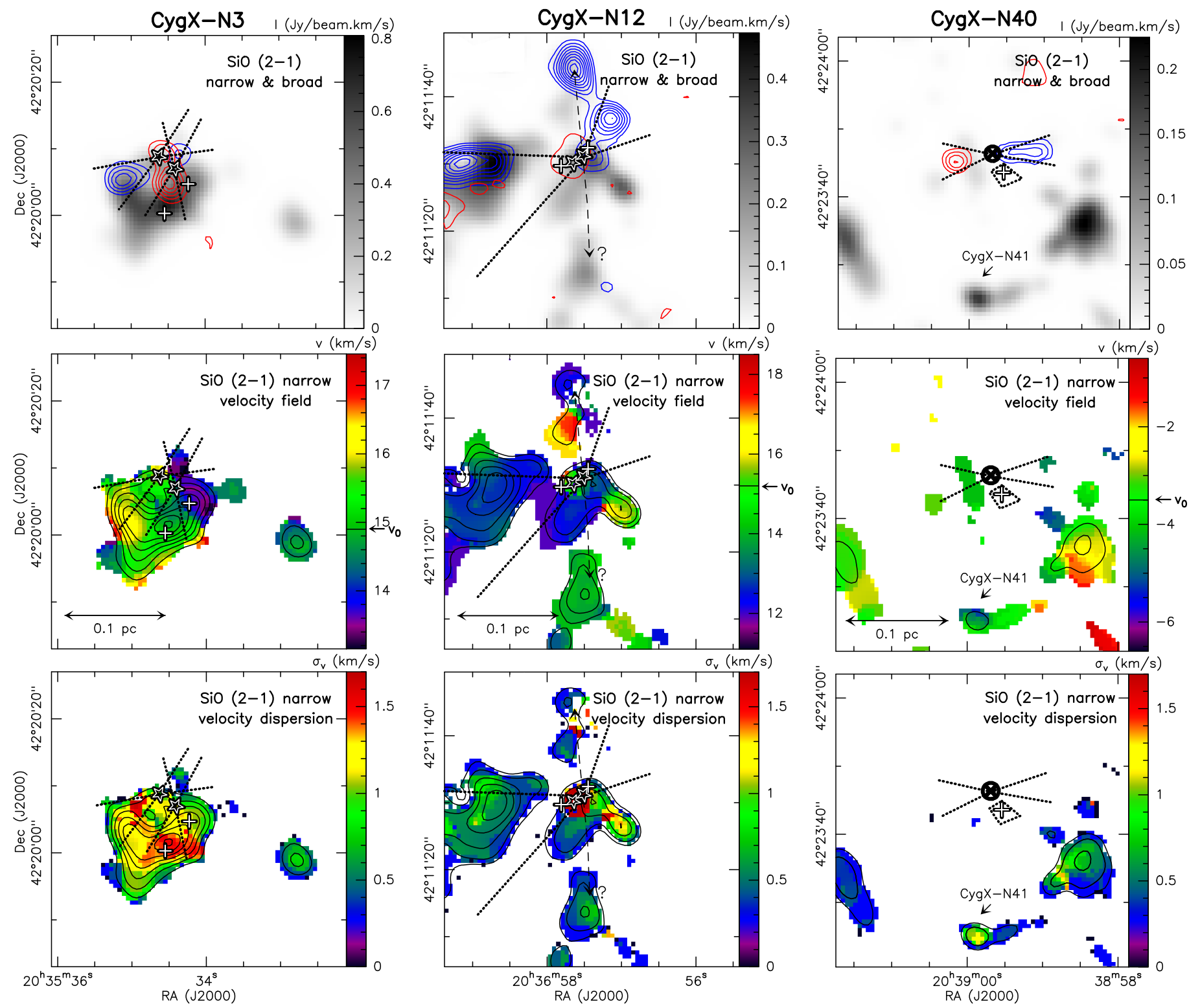

Fig. 4. Integrated intensity of the $\mathrm{SiO}$ emission at ambient velocities with the contribution from the broad outflow emission subtracted, for CygX-N3 (left), N12 (centre), and N40 (right). The top row panels show the narrow emission as greyscale, with contours from the high-velocity (broad) $\mathrm{SiO}$ emission from Fig. 2 overlaid. The two lower rows show the integrated narrow $\mathrm{SiO}$ emission in contours (with steps of $0.1 \mathrm{Jy} \mathrm{beam}^{-1} \mathrm{~km} \mathrm{~s}^{-1}$, starting at $0.1 \mathrm{Jy}_{\text {beam }}^{-1} \mathrm{~km} \mathrm{~s}^{-1}$ ), with the colour scale showing the respective moment maps: the velocity field (first moment map) in the middle row, and the velocity dispersion (second moment map) in the last row. Annotations are as in Fig. 2.

(integrated intensities, velocity field, and velocity dispersion) of the $\mathrm{SiO}$ emission at ambient cloud velocities as a result of this exercise. The three moments were calculated using the central $6 \mathrm{~km} \mathrm{~s}^{-1}$ velocity range, centred on the $v_{0}$ value given in Table 2 . Since the narrow component (as measured from the average spectra) has a $\sigma_{v} \lesssim 1.5 \mathrm{~km} \mathrm{~s}^{-1}$, this velocity range is enough to contain more than $95 \%$ of the narrow emission. Naturally, this method is only correct if/when the broad outflow emission is Gaussian, and when both narrow and broad components are well distinguished, but this is not always the case. Therefore, the resulting narrow emission maps may still show some residual broad emission (see the velocity dispersion maps of Figs. 4 and 5). For the purpose of these figures, we degraded slightly the spatial resolution of the integrated intensity maps (i.e. the zeroth-moment maps), by convolving with a Gaussian kernel of two arcseconds, merely so as to smooth the appearance of the contours, namely around individual pixels where the broad component was not successfully removed ${ }^{1}$. We did not do this spatial convolution for the velocity field and velocity dispersion maps, and the latter can be used to spot the positions where this method was not successful (pixels that still have large velocity dispersions, with $\left.\sigma_{v}>1.5 \mathrm{~km} \mathrm{~s}^{-1}\right)$.

In CygX-N3 (left column of Fig. 4), based on the location of the CO outflows, we have determined that about $60 \%$ of the total PdBI SiO luminosity is not constrained to the location of known outflows (as defined by the existence of $\mathrm{SiO}$ highvelocity emission). There is, therefore, a significant fraction of $\mathrm{PdBI} \mathrm{SiO}$ emission that is not clearly associated with any outflow, and which has narrow line profiles $\left(\sigma_{v} \sim 1.2 \mathrm{~km} \mathrm{~s}^{-1}\right.$, i.e. $F W H M \sim 2.8 \mathrm{~km} \mathrm{~s}^{-1}$ ). The velocity field of this narrow

1 This occurred in pixels where either the emission was not purely Gaussian, or the broad emission was too weak to pass our initial selection criteria for performing the broad Gaussian fitting. 

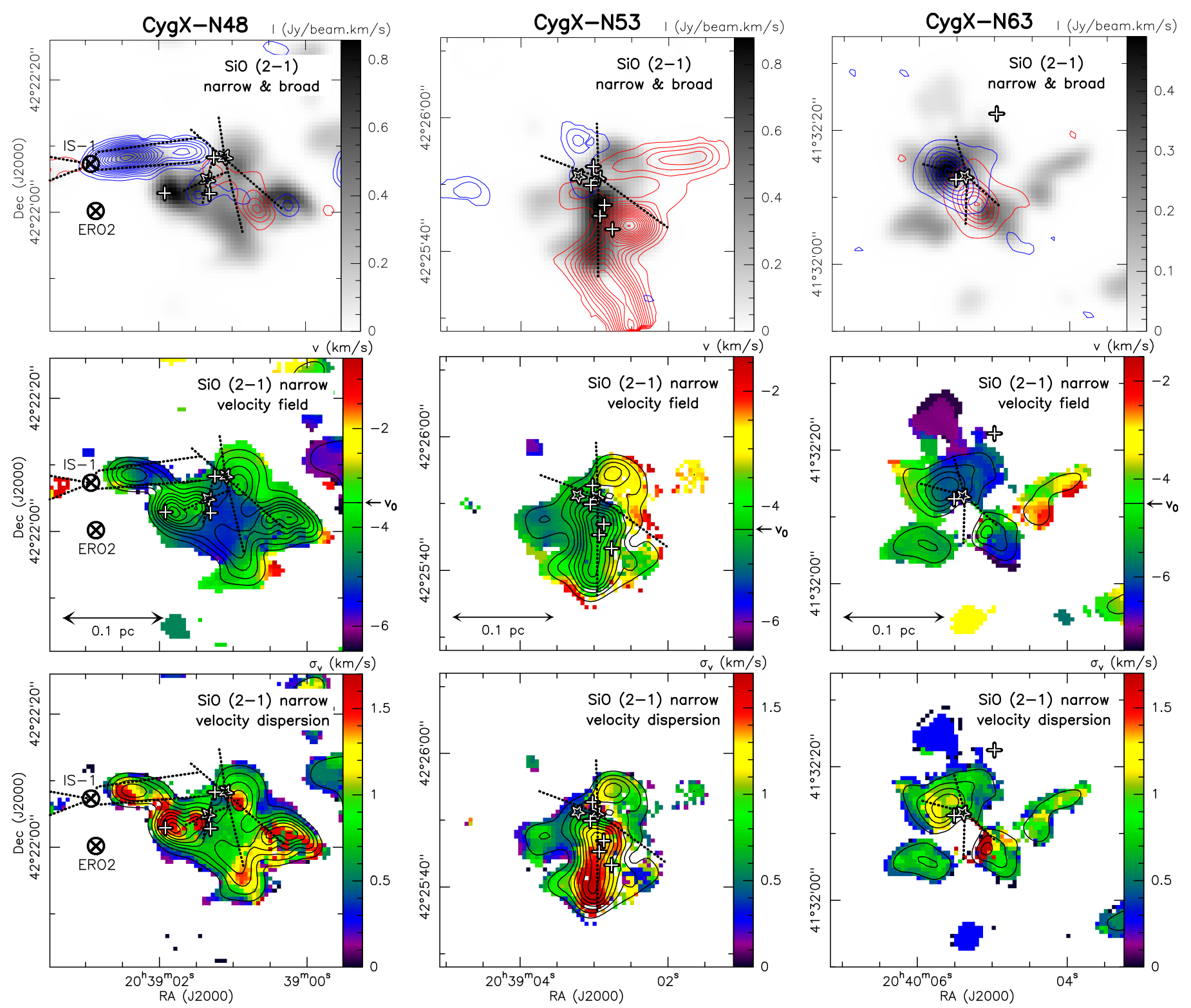

Fig. 5. Same as Fig. 4, for CygX-N48 (left), N53 (centre), and N63 (right). Top panels have the narrow SiO emission in greyscale and the highvelocity (broad) $\mathrm{SiO}$ emission in contours as in Fig. 3. The two lower rows show the integrated narrow $\mathrm{SiO}$ emission in contours (with steps of $0.1 \mathrm{Jy}_{\text {beam }}^{-1} \mathrm{~km} \mathrm{~s}^{-1}$, starting at $0.1 \mathrm{Jy} \mathrm{beam}^{-1} \mathrm{~km} \mathrm{~s}^{-1}$ ), with the respective moment maps in colour scale: the velocity field (first moment map) in the middle row, and the velocity dispersion (second moment map) in the last row. Annotations are as in Fig. 3.

component shows a large velocity gradient across the sources (of $2 \mathrm{~km} \mathrm{~s}^{-1}$ in $0.05 \mathrm{pc}$ ), with a shear-like structure aligned with the continuum filamentary structure. Interestingly, the velocity gradient seen in $\mathrm{SiO}$ is opposite to that of the $\mathrm{H}^{13} \mathrm{CO}^{+}$flows seen by Csengeri et al. (2011a, also with a velocity gradient of $\sim 2 \mathrm{~km} \mathrm{~s}^{-1}$ in $0.05 \mathrm{pc}$, but in the reverse direction; see their Fig. 11).

In CygX-N12 (central column of Fig. 4), $45 \%$ of the PdBI $\mathrm{SiO}$ luminosity arises from narrow low-velocity gas $\left(\sigma_{v} \sim\right.$ $0.8 \mathrm{~km} \mathrm{~s}^{-1}$, i.e. $F W H M \sim 1.8 \mathrm{~km} \mathrm{~s}^{-1}$ ). This includes the narrow blueshifted emission towards the southern edge of the blueshifted outflow lobe (see blue emission in the velocity field panel of Fig. 4, or narrow spectra from Fig. A.2), which accounts for the missed emission from the inner $30^{\prime \prime}$ but recovered in the $50^{\prime \prime}$ area. In this particular case, the narrow $\mathrm{SiO}$ emission is not spatially overlapping with an outflow and yet it is clearly associated with one, perhaps indicating that we are seeing the post-shock gas at the edges of an outflow cavity. The remaining ambient gas has a spatial distribution slightly extended along the N-S direction around the central sources, with a rather complex velocity field which makes it hard to compare with the (also complex) velocity field from $\mathrm{H}^{13} \mathrm{CO}^{+}$(Csengeri et al. 2011a).

For CygX-N40 (right column of Fig. 4), a significant amount of the total PdBI emission arises from systemic velocities $(54 \%)$, in the form of extended narrow emission, with a velocity dispersion reaching as low as $\sigma_{v} \sim 0.3 \mathrm{~km} \mathrm{~s}^{-1}$ (i.e. $\left.F W H M \sim 0.7 \mathrm{~km} \mathrm{~s}^{-1}\right)$. Some of the $\mathrm{SiO}$ emission is associated with DR21(OH)N1 (or CygX-N41 from Motte et al. 2007), seen as a $3 \mathrm{~mm}$ continuum peak to the south of MM1. The existence of negative side-lobes in SiO between MM1 and the arc-shaped emission around MM1 and MM2 means that despite recovering a good fraction of the emission observed with single-dish, the emission is quite extended and filtered out by the PdBI. Therefore, the fidelity of the imaging may not be very good (and the arc structure may not be real). Nevertheless, the existence of such emission is significant. It is not associated with the central cores, nor with outflows, and its origin is to be elucidated. 
Table 3. Source properties and estimated $\mathrm{SiO}$ luminosities.

\begin{tabular}{|c|c|c|c|c|c|c|c|c|c|}
\hline \multirow{3}{*}{ Source } & \multirow{3}{*}{$\begin{array}{c}\text { RA } \\
(\mathrm{J} 2000)\end{array}$} & \multirow{3}{*}{$\begin{array}{c}\text { Dec } \\
\mathrm{J}(2000)\end{array}$} & \multirow{3}{*}{$\begin{array}{l}M_{\text {env }} \\
\left(M_{\odot}\right)\end{array}$} & \multirow{3}{*}{$\begin{array}{c}L_{\text {bol }} \\
\left(L_{\odot}\right)\end{array}$} & \multirow{3}{*}{$\begin{array}{l}F_{\mathrm{CO}} \times 10^{-5}\left(M_{\odot}\right. \\
\left.\mathrm{km} \mathrm{s}^{-1} \mathrm{yr}^{-1}\right)\end{array}$} & \multicolumn{4}{|c|}{$L_{\mathrm{SiO}}\left(10^{8} \mathrm{~K} \mathrm{~km} \mathrm{~s}^{-1} \mathrm{pc}^{2}\right)$} \\
\hline & & & & & & \multicolumn{2}{|c|}{ Outflow emission } & \multicolumn{2}{|c|}{ Entire field } \\
\hline & & & & & & Total & High- $v$ & Total & Narrow \\
\hline N3-MM1 & $20: 35: 34.63$ & $42: 20: 08.8$ & $12.5 \pm 3.7$ & $106 \pm 60$ & 131 & 0.75 & $0.37(50 \%)$ & \multirow{2}{*}{3.85} & \multirow{2}{*}{$1.86(48 \%)$} \\
\hline N3-MM2 & 20:35:34.41 & 42:20:07.0 & $13.8 \pm 5.6$ & $121 \pm 50$ & 72 & 0.79 & $0.29(37 \%)$ & & \\
\hline N12-MM1 & $20: 36: 57.65$ & $42: 11: 30.2$ & $17.7 \pm 6.9$ & $485 \pm 130$ & $>36$ & \multirow{2}{*}{$2.65^{a}$} & \multirow{2}{*}{$1.39^{(a)}(52 \%)$} & \multirow{2}{*}{4.61} & \multirow{2}{*}{$2.07(45 \%)$} \\
\hline N12-MM2 & $20: 36: 57.51$ & $42: 11: 31.2$ & $16.4 \pm 5.8$ & $195 \pm 75$ & $>12$ & & & & \\
\hline N40-MM1 & 20:38:59:54 & $42: 23: 43.6$ & $1.9 \pm 0.5$ & - & 7 & - & - & \multirow{2}{*}{3.29} & \multirow{2}{*}{$1.77(54 \%)$} \\
\hline N40-MM2 & 20:38:59:69 & $42: 23: 46.3$ & $1.0 \pm 0.5$ & - & 28 & 0.33 & $0.29(88 \%)$ & & \\
\hline N48-MM1 & 20:39:01.34 & $42: 22: 04.9$ & $17.0 \pm 6.4$ & $102 \pm 70$ & 135 & 0.99 & $0.89(90 \%)$ & \multirow{2}{*}{$11.84^{b}$} & \multirow{2}{*}{$4.6(38 \%)$} \\
\hline N48-MM2 & 203901.10 & 422208.3 & $8.1 \pm 3.0$ & $85 \pm 60$ & 45 & 2.00 & $0.51(26 \%)$ & & \\
\hline N53-MM1 & 20:39:02.96 & $42: 25: 51.0$ & $34.2 \pm 11.1$ & $199 \pm 70$ & 412 & 10.34 & $7.58(73 \%)$ & \multirow{2}{*}{11.57} & \multirow{2}{*}{$2.06(18 \%)$} \\
\hline N53-MM2 & 20:39:03.22 & $42: 25: 51.2$ & $21.4 \pm 6.1$ & $144 \pm 50$ & $<121$ & $<0.23$ & $<0.11(48 \%)$ & & \\
\hline N63-MM1 & 20:40:05.39 & 42:32:13.1 & $44.3 \pm 11.9$ & $339 \pm 50$ & 291 & 4.89 & $3.49(71 \%)$ & 5.93 & $1.08(18 \%)$ \\
\hline
\end{tabular}

Notes. ${ }^{(a)}$ With the resolution of our $\mathrm{SiO}$ maps and the configuration of the outflows in N12, we cannot distinguish the outflow from the two millimetre sources. The values presented are for the combination of the two. ${ }^{(b)}$ The total SiO luminosity in N48 includes the high-velocity outflow from IS-1 and a high velocity blue and red emission to the west, each of which account for $20 \%$ of the total SiO luminosity.

For CygX-N48 (left column of Fig. 5), even though most of the PdBI SiO luminosity is associated with low-velocity gas $(\sim 70 \%)$, there is a significant spatial overlap of the narrow $\mathrm{SiO}$ emission with high-velocity $\mathrm{SiO}$, suggesting that a fraction of the low-velocity $\mathrm{SiO}$ emission could be associated with the outflows of the region (e.g. the emission associated with the IS-1, MM1, and/or MM2 outflows). Nevertheless, there is some narrow $\mathrm{SiO}$ emission which lies outside outflow areas $(\sim 20 \%)$. The origin of this emission could be associated with the low-velocity shocks detected in this region by Csengeri et al. (2011b) in $\mathrm{N}_{2} \mathrm{H}^{+}$ (see Sect. 4.2.2 below).

In CygX-N53 (central column of Fig. 5) there is only a relatively small amount of PdBI SiO emission at systemic velocities $(\sim 35 \%)$. This emission shows a SE-NW elongation that has a velocity gradient of $2 \mathrm{~km} \mathrm{~s}^{-1}$ in $0.1 \mathrm{pc}$, with a direction reminiscent of the rotation of the MDC and the envelope (as seen in $\mathrm{H}^{13} \mathrm{CO}^{+}$; see Fig. 5 of Csengeri et al. 2011a). The narrow $\mathrm{SiO}$ emission to the south, however, could be tracing the lower-velocities of the redshifted outflowing gas.

Finally, in CygX-N63 (right column of Fig. 5), the narrow emission is broken up in two areas. One to the north of (and including) MM1, which shows a velocity gradient across MM1 (E-W), with a direction consistent with that seen in $\mathrm{H}^{13} \mathrm{CO}^{+}$ (Csengeri et al. 2011a). This emission is coincident with the blueshifted outflow lobe, and the sharp velocity gradient of $\sim 2 \mathrm{~km} \mathrm{~s}^{-1}$ in $0.05 \mathrm{pc}$ is coincident with the eastern edge of the lobe, perpendicular to the direction of the outflow. This could indicate that the $\mathrm{SiO}$ is tracing the cavity walls of the outflow. Farther south, there is another E-W extended emission that has narrow linewidths $\left(\sigma_{v} \sim 0.8 \mathrm{~km} \mathrm{~s}^{-1}\right.$, i.e. $\left.F W H M \sim 1.8 \mathrm{~km} \mathrm{~s}^{-1}\right)$. While the central peak could be interpreted as being part of the redshifted outflow walls, the origin of the extensions to the east and west are less clear.

\subsection{Narrow SiO column densities}

To estimate the order of magnitude of the column densities of the $\mathrm{SiO}$ narrow component, we have used the IRAM $30 \mathrm{~m}$ spectra of $\mathrm{SiO}$ (from Motte et al. 2007). Since these spectra include a contribution from broad outflow emission, we performed double Gaussian fittings to extract the narrow component.
The $\mathrm{H}_{2}$ volume densities in the MDCs are of the order of $\sim 10^{6}-10^{7} \mathrm{~cm}^{-3}\left(\sim 10^{6} \mathrm{~cm}^{-3}\right.$ being the average MDC densities, while $10^{7} \mathrm{~cm}^{-3}$ is more representative of the average density of the protostellar envelopes; see e.g. Louvet et al. 2014), which means that we are above the $\mathrm{SiO}$ critical densities, and hence we can assume LTE to estimate the column densities (as e.g. in Nguyên-Lu'o'ng et al. 2013). Assuming a temperature of 20-40 K, we estimate that the observed beam-averaged $\mathrm{SiO}$ integrated intensities for the narrow component correspond to $\mathrm{SiO}$ column densities of the order of $0.1-4 \times 10^{12} \mathrm{~cm}^{-2}$, where the optical depths are largely below one. We note, however, that this corresponds to the beam-averaged $\mathrm{SiO}$ column densities, within the IRAM $30 \mathrm{~m}$ beam. From the PdBI, we can see that the emission is more compact than the $30^{\prime \prime}$ beam, and therefore these column densities are merely a lower limit for the effective column densities. The PdBI emission having linear dimensions typically of the order of $\sim 15^{\prime \prime}$ (i.e. $\sim 0.1 \mathrm{pc}$ ) implies that the beam filling factors are easily low enough to bring the column densities to values closer to $\sim 10^{13} \mathrm{~cm}^{-2}$. Since the $\mathrm{H}_{2}$ column densities in the MDCs range between $6 \times 10^{23} \mathrm{~cm}^{-2}$ and $2 \times 10^{24} \mathrm{~cm}^{-2}$, we retrieve $\mathrm{SiO}$ abundances of the order of $0.5-1.0 \times 10^{-11}$ for the narrow $\mathrm{SiO}$ emission (which are similar to the abundance values of narrow $\mathrm{SiO}$ emission found in IRDCs by, e.g. Jiménez-Serra et al. 2010; Sanhueza et al. 2013).

\section{Analysis}

\subsection{SiO outflow luminosity}

To investigate the relation between the $\mathrm{SiO}$ luminosity, $L_{\mathrm{SiO}}$, and the respective $\mathrm{CO}$ outflow momentum flux, $F_{\mathrm{CO}}$, we have estimated the $L_{\mathrm{SiO}}$ associated with a given outflow, whenever possible. In practice, this involved the usage of polygons to limit the areas where to estimate the $\mathrm{SiO}$ flux. These areas were determined based on the extent and morphology of the $\mathrm{SiO}$ emission, but with the help of the $\mathrm{CO}$ emission presented in Duarte-Cabral et al. (2013), as the CO observations allow a more accurate identification of the driving sources of the different outflow lobes. We estimated this $L_{\mathrm{SiO}}$ for each source, first using the total velocity range, and then by restraining the calculations to the highvelocity $\mathrm{SiO}$ emission.

Table 3 summarises the results from this exercise, for the sample of sources for which we have been able to individually 


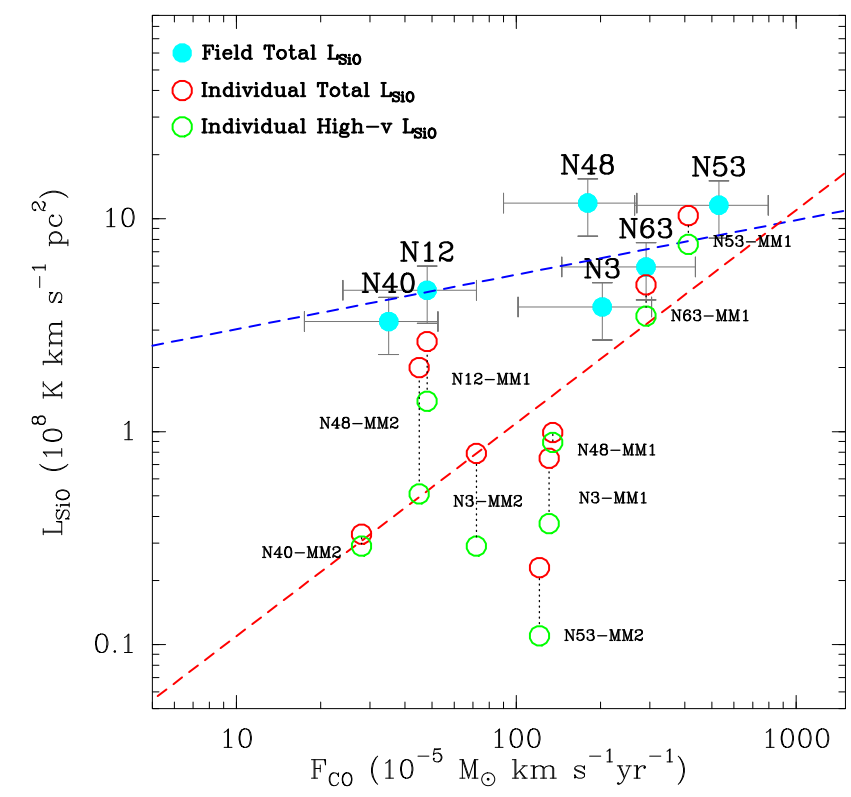

Fig. 6. Observed $L_{\mathrm{SiO}}$ against the corresponding $F_{\mathrm{CO}}$ (from Duarte-Cabral et al. 2013). The light blue filled circles correspond to the total $L_{\mathrm{SiO}}$ and total $F_{\mathrm{CO}}$ for each of the six fields, and the blue dashed line is the respective linear fit in a log-scale. The open circles are for the resolved individual outflows (integration spatially constrained to location of the individual outflows), with the $F_{\mathrm{CO}}$ estimated in Duarte-Cabral et al. (2013; Table 3, Col. 6). The red open circles have $L_{\mathrm{SiO}}$ estimated using the entire velocity range (Table 3, Col. 7), and the respective linear fit to the log-values is shown as a dashed red line, which translates into a linear correlation of $L_{\mathrm{SiO}} \propto F_{\mathrm{CO}}$. The green open circles show the $L_{\mathrm{SiO}}$ only from the outflow high-velocity wing emission (Table 3, Col. 8).

estimate the $\mathrm{SiO}$ luminosity. Columns 1 to 6 indicate the source names and positions, the envelope mass and bolometric luminosities retrieved from SED fittings, and the CO outflow momentum flux (the last three are from Duarte-Cabral et al. 2013). Columns 7 and 8 show the SiO luminosities associated with individual outflows, and the second-to-last column shows the total $\mathrm{SiO}$ luminosity in the entire PdBI field (equivalent to the integrated $\mathrm{SiO}$ emission that would be observed over the same field by a single-dish telescope). The last column shows the $\mathrm{SiO}$ luminosity in the entire PdBI field that arises from narrow emission (i.e. calculated from the integrated intensity maps shown in Figs. 4 and 5).

The first result from this estimate demonstrates that there is no $1: 1$ relation between $L_{\mathrm{SiO}}$ and $F_{\mathrm{CO}}$. Nevertheless, the two stronger outflows in the sample (from the two most massive sources, CygX-N53 MM1 and CygX-N63 MM1) are also those showing the most luminous $\mathrm{SiO}$ emission. This is illustrated well in Fig. 6, where we can see that the two stronger outflows (with $F_{\mathrm{CO}}>2 \times 10^{-3} M_{\odot} \mathrm{km} \mathrm{s}^{-1} \mathrm{yr}^{-1}$ ) also have strong SiO luminosities (both when taking the entire fields, and the individual sources). However, for the remaining fields, a correlation is less evident. When measuring the $\mathrm{SiO}$ luminosities on entire fields, which in essence mimics observations of $\mathrm{SiO}$ that do not resolve individual outflows, we see no correlation of $\mathrm{SiO}$ luminosity with the corresponding outflow power (the dashed blue line in Fig. 6 shows the tentative fit to the light blue circles, with a weak dependency of $L_{\mathrm{SiO}} \propto F_{\mathrm{CO}}^{\alpha \leqslant 0.3}$ ). When we are able to distinguish individual outflows, then the $\mathrm{SiO}$ luminosities do appear to correlate linearly with the outflow momentum fluxes measured

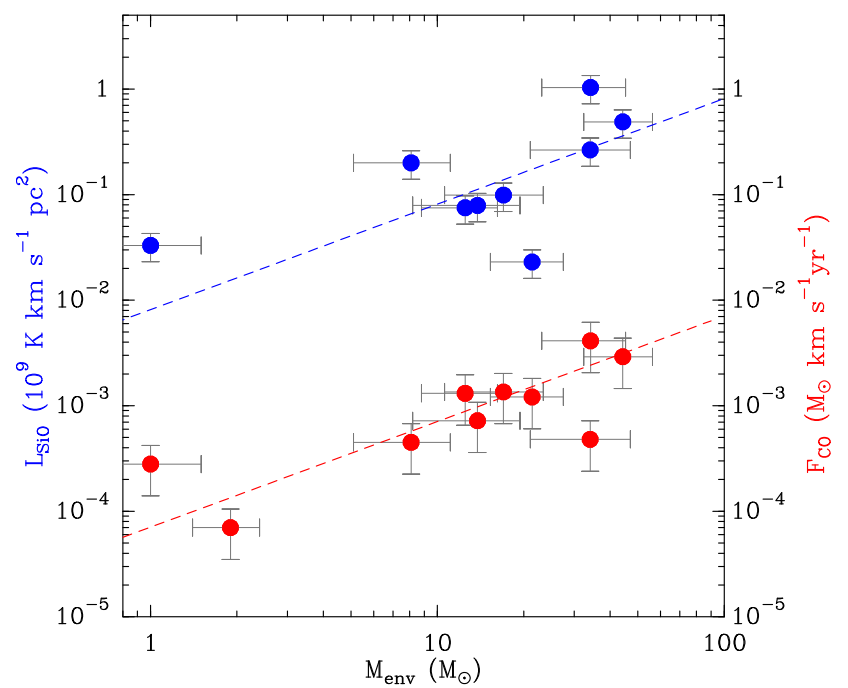

Fig. 7. Observed $L_{\mathrm{SiO}}$ (same as the red open circles in Fig. 6) and $F_{\mathrm{CO}}$, in blue and red circles, respectively, with respect to the envelope masses $\left(M_{\text {env }}\right)$ for individual sources. The second red point from the left corresponds to CygX-N40 MM1, for which we have an $F_{\text {CO }}$ measurement, but no $L_{\mathrm{SiO}}$. The dashed red line shows the linear correlation of $F_{\mathrm{CO}}=7.5 \times 10^{-5} M_{\mathrm{env}}$, originally found by Bontemps et al. (1996) for low-mass protostars, and confirmed in Duarte-Cabral et al. (2013) to extend to the high-mass regime. The dashed blue line shows the linear correlation of $L_{\mathrm{SiO}} \simeq 5 \times 10^{6} M_{\text {env }}$ that we find for the blue points. This figure accentuates the fact that $L_{\mathrm{SiO}}$ of individual outflows follows the same trend as $F_{\mathrm{CO}}$ (both being $\propto M_{\text {env }}$ ), even though it introduces more scatter with respect to the linear relation found for $F_{\mathrm{CO}}$.

from $\mathrm{CO}$ lines (see dashed red line in Fig. 6, which corresponds to $L_{\mathrm{SiO}} \propto F_{\mathrm{CO}}$ ). This result suggests that $\mathrm{SiO}$ luminosities associated with individual outflows could statistically be used as a proxy for the outflow power. However, as shown in Table 3, there is a significant scatter of more than one order of magnitude for the $\mathrm{SiO}$ luminosities, for similar values of $F_{\mathrm{CO}}$. The larger scatter in $L_{\mathrm{SiO}}$ is also visible in Fig. 7 , where we show the relation between both $L_{\mathrm{SiO}}$ (in blue) and $F_{\mathrm{CO}}$ (in red) with the $M_{\mathrm{env}}$, for the protostars of our sample. This figure shows that both $L_{\mathrm{SiO}}$ and $F_{\mathrm{CO}}$ scale linearly with envelope mass, when individual outflows can be resolved, in spite of the larger apparent scatter from the $L_{\mathrm{SiO}}$. We caution that these results are based on our small sample, and need to be confirmed with larger samples.

\subsection{Nature of the narrow $\mathrm{SiO}$ emission}

The fraction of $\mathrm{SiO}$ emission at low velocities is highly variable (Table 3), and while in regions with strong outflows (e.g. CygX-N53 and CygX-N63) the SiO high-velocity emission accounts for $80 \%$ of the full $\mathrm{SiO}$ luminosity, other sources have the inverse situation, where most of the $\mathrm{SiO}$ emission arises from narrow emission at systemic velocities. Such emission could be due to shocks other than outflows. To help clarify the nature of the narrow $\mathrm{SiO}$ emission, we summarise the results from Sect. 3.3 in Table 4, by describing the emission in the regions according to four distinct cases: (1) the existence of spatially overlapping broad and narrow emission; (2) the existence of broad emission with little/no narrow emission associated; (3) the existence of narrow emission adjacent to (but not coincident with) broad emission; and (4) the existence of narrow emission with absolutely no broad emission in the vicinity. We will look in more detail into each case in the following sections. 
A. Duarte-Cabral et al.: $\mathrm{SiO}$ emission from low- and high-velocity shocks in Cygnus-X massive dense clumps

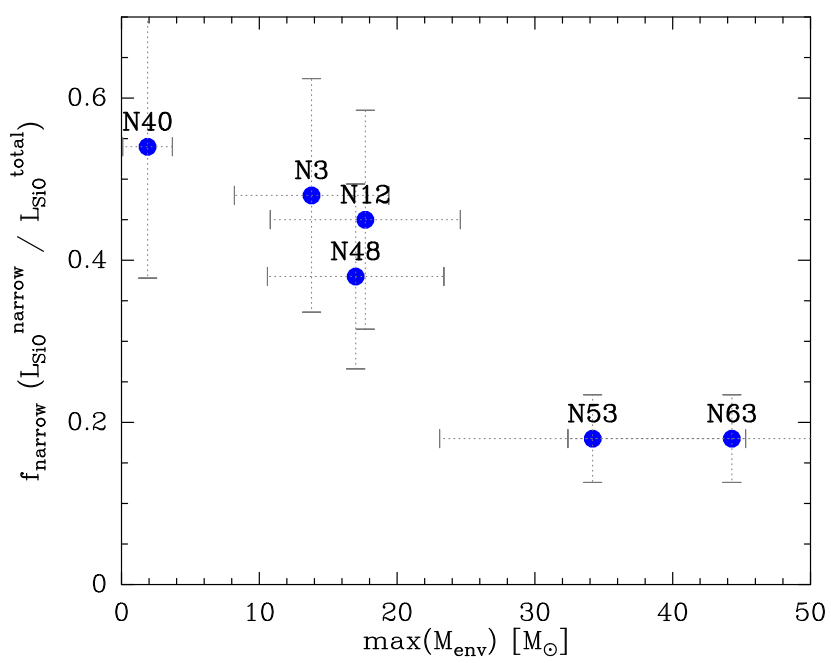

Fig. 8. Fraction of the total $\mathrm{SiO}$ luminosity that arises from the narrow component, $f_{\text {narrow }}$, as a function of the mass of the most massive protostellar envelope which is currently forming in each MDC. This plot accentuates the trend of a higher fraction of the emission in the form of narrow emission for the MDCs which have not yet gathered most of their mass in the envelopes of the forming protostars.

\subsubsection{Narrow $\mathrm{SiO}$ emission from outflows}

Strong high-velocity outflows (as those powered by the two most massive sources CygX-N53 and CygX-N63), have naturally a strong impact on the ambient gas, and can easily form and entrain $\mathrm{SiO}$ to high velocities. There are, however, several factors that come into play when $\mathrm{SiO}$ is formed in such a high-velocity shock, and these factors can change the observed line profile.

The typical profile of $\mathrm{SiO}$ produced in high-velocity shocks has a highly asymmetric profile, including a broad and a narrow component (from 1D models, e.g. Gusdorf et al. 2008a; Anderl et al. 2013). This typical profile explains case (1) of Table 4, where broad and narrow emission are spatially overlapping. While such models predict the narrow profiles to be shifted by tens of $\mathrm{km} \mathrm{s}^{-1}$ from the systemic velocities, projection effects can contribute to significantly decrease the observed velocity shifts of the shocked gas with respect to the ambient velocities. On the other hand, projection effects can also have another consequence, which is the fact that the profile observed is a result of the superposition of several ideal $1 \mathrm{D}$ profiles. One of the results of this can be the symmetrisation of the asymmetric profiles, i.e. the narrow component will become less obvious and the emission we see is mostly a broad (near Gaussian) profile. This explains the profiles of case (2) of Table 4.

If, however, the composition of the pre-shock gas is such that the silicon is solely in the grain cores, the high-velocity shocks will produce $\mathrm{SiO}$ with narrower profiles (Gusdorf et al. 2008a; Anderl et al. 2013). Therefore, the existence of narrow emission offset from the systemic velocities, with no broad emission associated, might be the signature of a fraction of $\mathrm{SiO}$ as emanating from the grain cores. Another way to have narrow $\mathrm{SiO}$ emission associated with high-velocity shocks, is as a result of the mixing of the shocked gas with the colder ambient gas, which can still form $\mathrm{SiO}$ but with lower velocity dispersion (e.g. Lefloch et al. 1998; Codella et al. 1999). Even though the timescales for the deceleration of the shocked gas or alternatively the oxidisation of $\mathrm{SiO}$ or its depletion back into the grain ice mantles are short (both of the order of $\sim 10^{4} \mathrm{yr}$, e.g. Martin-Pintado et al. 1992; Codella et al. 1999), the high-mass protostars in
Table 4. Distribution of the $\mathrm{SiO}(2-1)$ emission.

\begin{tabular}{l|l}
\hline \hline Field & Configuration \\
\hline CygX-N3 & $(1),(3),(4)$ \\
CygX-N12 & $(1),(3),(4)$, marginal (2) in the NW of the cone \\
CygX-N40 & $(2),(4)$, marginal (3) near the red lobe \\
CygX-N48 & $(1),(3)$, marginal (4) between southbound outflows \\
CygX-N53 & $(1),(2),(3)$, marginal (4) to the SE of MM2 \\
CygX-N63 & $(1),(3),(4)$ \\
\hline
\end{tabular}

Notes. (1) Spatial overlap between broad and narrow emission. (2) Broad emission without narrow emission. (3) Narrow emission with nearby broad emission. (4) Narrow emission with no broad emission in the vicinity.

these MDCs are Class 0 equivalents, and therefore also relatively young. Furthermore, the outflows being a continuous process, they have the potential to continuously replenish the decaying decelerated $\mathrm{SiO}$ in outskirts of outflows. In this case, we expect to see narrow $\mathrm{SiO}$ emission at systemic velocities, in the vicinity of high-velocity shocks, for example, along outflow walls. Alternatively, and as suggested by Jiménez-Serra et al. (2004), the presence of narrow $\mathrm{SiO}$ at systemic velocities could also be explained by recent sputtering of the grain mantles containing a small fraction of $\mathrm{Si} / \mathrm{SiO}$ and therefore could be indicative of young shocks (or simply tracing of the most recently shocked material). All three options could explain configuration (3) of Table 4.

Finally, another way that we could achieve narrow profiles from a high-velocity shock would be with a strong UV field from the protostar, illuminating either the pre- or post-shock region, effectively decreasing the thickness of the $\mathrm{SiO}$ emitting-region, and subsequently narrowing its line profiles (Gusdorf et al., in prep.). However, our sample of protostars (some of which host hot cores, e.g. CygX-N53 MM1 and CygX-N63 MM1) are Class 0 equivalents (with $L_{\text {bol }} \sim 100-500 L_{\odot}$, see Duarte-Cabral et al. 2013, and Table 3), and therefore the radiation from the forming protostars is not yet strong enough to ionise the gas outside the inner protostellar envelopes/hot-core regions (which are unresolved by our observations), ruling out this hypothesis.

In our study, we do find narrow $\mathrm{SiO}$ line profiles associated with high-velocity outflow shocks. On one hand, we have narrow $\mathrm{SiO}$ emission at systemic velocities close to high-velocity outflow lobes, which could be due to the mixing of shocked gas and ambient gas along outflow walls. The most obvious candidates for this specific case in our sample are CygX-N40, next to the red outflow lobe; CygX-N53, along the red outflow cone, and to the west of the blue outflow lobe; and CygX-N63, around the blue outflow lobe, and at the tip of the red outflow. In CygX-N48 this process can also be in play, for instance along the edges of the red outflow from MM2 and next to the outflow from MM1. On the other hand, we also detected narrow $\mathrm{SiO}$ emission offset from systemic velocities, which could be an indication of a specific environment where the $S i$ is in grain cores. This is the case of CygX-N12, where there are narrow line profiles $\left(F W H M \sim 1 \mathrm{~km} \mathrm{~s}^{-1}\right)$ associated with the east-bound outflow (see Fig. A.2) and slightly blueshifted from the systemic velocities.

\subsubsection{Other narrow emission}

Despite the existence of $\mathrm{SiO}$ narrow emission associated with outflows, we also detect some narrow $\mathrm{SiO}$ emission, at systemic 
velocities, that does not have any broad outflow emission in the immediacies. This is our configuration (4).

This is most striking for CygX-N40 where most of the $\mathrm{SiO}$ emission is narrow, extended, and offset (by $\gtrsim 0.1 \mathrm{pc}$ ) from the forming protostars, hence definitely not associated with outflows $(\lesssim 0.05 \mathrm{pc})$ nor any hot-core $(<0.005 \mathrm{pc})$. For this specific region, we have also investigated whether this extended $\mathrm{SiO}$ emission could be due to external radiation, but this seems unlikely as we have found no evidence of higher dust temperatures surrounding the N40 core (see e.g. Hennemann et al. 2012). It is perhaps worth noting that this region sits close to the convergence point between the DR21 ridge and another filament thought to be feeding material into the main ridge (Schneider et al. 2010). The shocks from this infall of material into the MDC could explain the existence of extended $\mathrm{SiO}$ emission (in line with what had been interpreted for other regions with extended narrow $\mathrm{SiO}$ emission with FWHM of $0.8-2 \mathrm{~km} \mathrm{~s}^{-1}$, e.g. by Jiménez-Serra et al. 2010; Nguyên-Lu'o'ng et al. 2013; Sanhueza et al. 2013).

In CygX-N3 and N48, even though the narrow low-velocity emission has a significant overlap/adjacency to outflow regions, the $\mathrm{SiO}$ narrow emission has a similar spatial distribution to the interface regions of the small-scale converging flows observed here (Csengeri et al. 2011a,b), suggesting that the $\mathrm{SiO}$ could also have been formed from the low-velocity shocks of such flows. Interestingly, in both cases, the $\mathrm{SiO}$ narrow emission has a velocity gradient which does not mimic the velocity shears seen in high-density tracers $\left(\mathrm{H}^{13} \mathrm{CO}^{+}\right.$and $\mathrm{N}_{2} \mathrm{H}^{+}$, Csengeri et al. 2011a,b). The mismatched velocity field could be explained by the fact that the $\mathrm{SiO}$ is not formed on the flows themselves, but as part of the post-shock material. If this post-shock material is originally at systemic velocities, and is dense enough to retain its original velocity field despite the external flow, the $\mathrm{SiO}$ would retain the original velocity of the (denser) local gas.

In Cyg-X N48, Csengeri et al. (2011b) detected $\mathrm{CH}_{3} \mathrm{CN}$ as well, and suggest that it is tracing the gas shocked by such converging low-velocity flows. In Fig. 9 we show how the narrow $\mathrm{SiO}$ emission (in contours) correlates with this $\mathrm{CH}_{3} \mathrm{CN}$ emission. Some of the $\mathrm{CH}_{3} \mathrm{CN}$ emission could be associated with high-velocity outflows as they peak in regions adjacent to outflow lobes (e.g. the peak next to the outflow from IS-1). Nevertheless, it is interesting to note that the interface between the two converging flows (marked with a blue line) coincides with the most massive protostar of this MDC, and could be responsible for the high star formation activity in this region. This interface has both $\mathrm{CH}_{3} \mathrm{CN}$ and narrow $\mathrm{SiO}$ emission, supporting the idea that they could both potentially arise not only from the several high-velocity outflows, but also from the shocks from small-scale converging flows.

In CygX-N12, CygX-N53, and CygX-N63, apart from the narrow emission that is associated with outflows (either blueshifted or in outflow cavity walls), there are small extensions with narrow emission at systemic velocities where no outflows co-exist. This $\mathrm{SiO}$ narrow emission extensions in CygX-N53, N63, and N12 could, similarly to N3 and N48, be pinpointing the convergence zones of the global collapse of the MDC material (or perhaps even the collapse of larger scale cloud structures) onto the protostellar envelopes.

\section{Discussion and conclusions}

\subsection{SiO as a probe of outflow shocks}

Our study confirms that $\mathrm{SiO}$ is a good indicator of outflows in massive star-forming regions. However, we advise caution when

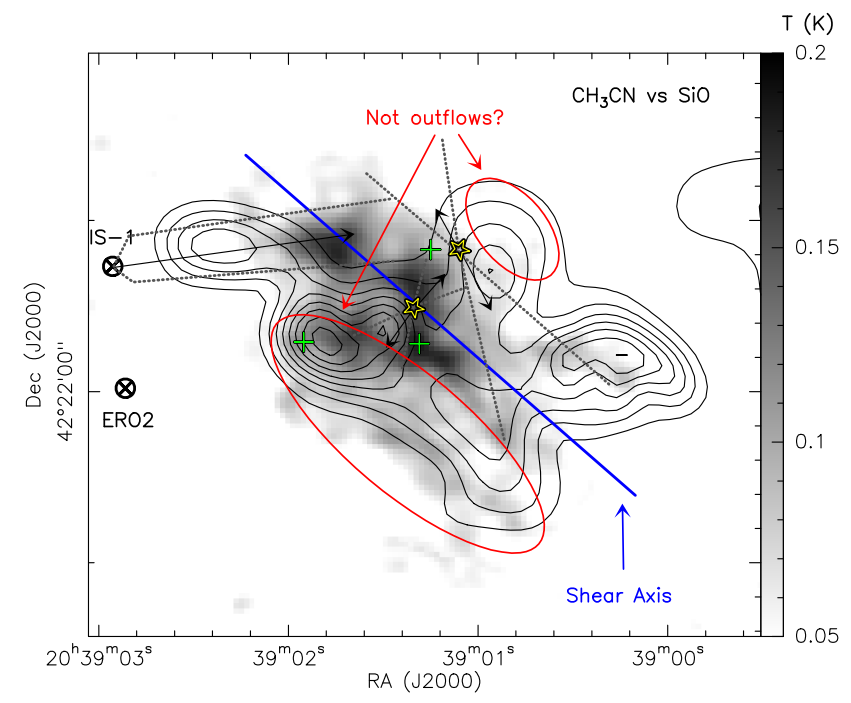

Fig. 9. Map of the CygX-N48 integrated intensity of $\mathrm{CH}_{3} \mathrm{CN}$ from (Csengeri et al. 2011b) in greyscale, overlaid with the $\mathrm{SiO}$ systemic velocity emission from this paper in contours (for both molecules the velocity integration range was from -6.5 to $-0.5 \mathrm{~km} \mathrm{~s}^{-1}$ ). The red ellipses show the regions with narrow $\mathrm{SiO}$ emission adjacent to the clear velocity shear found in $\mathrm{N}_{2} \mathrm{H}^{+}$, shown here as a blue line. Even though there are strong and compact $\mathrm{SiO}$ peaks coincident with high-velocity emission as seen in Fig. 5, the weaker and more extended $\mathrm{SiO}$ systemic velocity emission could be associated with the lower-velocity shocks from this velocity shear.

using the $\mathrm{SiO}$ luminosity as a proxy of outflow power when individual outflows cannot be resolved. Doing so in our sample of sources would have induced an overestimation of the outflow power which could reach more than an order of magnitude (e.g. in CygX-N3, N40, and N48). This is mostly due either to the existence of a narrow component of $\mathrm{SiO}$ emission, often extended, which may not be due to outflow shocks. Nevertheless, the stronger the outflows, the more the outflow SiO luminosity will dominate over any other contributing $\mathrm{SiO}$ emission, resulting in a tighter correlation with outflow power.

These problems are attenuated when we are able to separate the individual outflows, where we tentatively recover a linear relation between $F_{\mathrm{CO}}$ and the total $L_{\mathrm{SiO}}$, but because $\mathrm{SiO}$ needs to be formed and entrained by the outflows (unlike $\mathrm{CO}$ that needs only to be entrained), the $\mathrm{SiO}$ outflow emission depends not only on the efficiency of the outflows in forming and entraining $\mathrm{SiO}$ (which is likely linked to the outflow power and, as such, the evolutionary stage of the protostar), but it also depends on the amount of material encountered and shocked by the outflows along their propagating axis as they pierce the cloud. The scatter is therefore more significant for the relation of $\mathrm{SiO}$ luminosity with envelope mass, than it is when using the $\mathrm{CO}$ outflow momentum fluxes.

\subsection{Origin of the narrow $\mathrm{SiO}$ emission}

From our sample of sources, we have realised that MDCs forming the least massive stars have their SiO luminosity dominated by a narrow component of $\mathrm{SiO}$ at systemic velocities (see Fig. 8). This narrow component, however, does seem to exist in all cases. While for the most massive cores it seems mostly related to outflow walls or compact narrow emission close to the protostellar envelopes (at systemic velocities), the least massive sources have a tendency to show more extended $\mathrm{SiO}$ narrow emission. Such 
narrow $\mathrm{SiO}$ emission could have various origins, and in the following we shall explore some of the possibilities.

One hypothesis is that it arises from the mixing of the gas due to the interaction of different outflows from a population of low-mass stars undetected in the PdBI continuum images. We note, however, that the sensitivity in the $1 \mathrm{~mm}$ continuum observations is equivalent to $\sim 1 M_{\odot}$, and that all the potential lowmass protostars with envelope masses above $\sim 1 M_{\odot}$ are listed by Bontemps et al. (2010) and shown as crosses in our figures. The positions of these candidate low-mass protostars are not associated with any $\mathrm{CO}$ high-velocity outflow (which we would expect to detect as we did in CygX-N40), and they also do not lie near most of the narrow emission detected. Judging by the number of low-mass fragments detected we do not expect a significant underlying population of very low-mass protostars $\left(<1 M_{\odot}\right)$. Even though we cannot rule out this hypothesis completely, we consider that this scenario is thus unlikely.

Another hypothesis is that the shocks that may create the narrow $\mathrm{SiO}$ emission where no nearby high-velocity shocks exist could be related to converging flows shocking with the cloud's material. In our sample, these shocks could be due to the smallscale converging flows reported by Csengeri et al. (2011a,b) or due to the large scale collapse of the DR21 ridge as suggested by Schneider et al. (2010). This is in line with what had been suggested for other high-mass star-forming regions by e.g. Jiménez-Serra et al. (2010); Nguyên-Lu'o'ng et al. (2013). Perhaps seeing a dominant $\mathrm{SiO}$ narrow emission is actually a sign that there is a significant and ongoing infall of material from beyond the MDC scale $(\gtrsim 0.1-0.3 \mathrm{pc})$ to the envelope scale $(\lesssim 0.02 \mathrm{pc})$, which could lead to a further increase of the masses of existing individual cores or to the formation of new ones. The most striking case of our sample is undoubtedly CygX-N40 where the inner envelope is not yet massive at all, but there are $\mathrm{SiO}$ shocks everywhere around it. We note that in spite of comprising nearly $\sim 100 M_{\odot}$ inside less than $0.15 \mathrm{pc}$ (Motte et al. 2007), Cygx-N40 has a rather shallow density profile, with no high-mass protostars yet formed in the centre. Perhaps N40 is at an earlier stage than the other sources, where the material of the MDC is yet to become centrally condensed and form one or more high-mass protostellar cores, and the $\mathrm{SiO}$ emission is the evidence of the shocks of the infall of large-scale flows and the collapsing MDC itself.

One of the problems with having $\mathrm{SiO}$ at low velocities not associated with outflows is to understand if and how low-velocity shocks could form $\mathrm{SiO}$. As mentioned by Nguyên-Lu'o'ng et al. (2013), SiO can be efficiently formed by low-velocity shocks $\left(<10 \mathrm{~km} \mathrm{~s}^{-1}\right)$ provided that there is already some $\mathrm{Si}$ in the gas phase or in ice mantles. Having Si surviving in the gas phase in the cold and dense environment of an MDC is, however, not very likely since $\mathrm{Si}$ is expected to either deplete back onto the grain ice mantles, or to be oxidised in $\mathrm{SiO}_{2}$, itself being subsequently depleted back onto the grain ice mantles, both processes leading to its disappearance from the gas phase in about $\sim 10^{4}$ yr (e.g. Martin-Pintado et al. 1992; Codella et al. 1999; Gusdorf et al. 2008a). Nevertheless, it is possible to admit that the converging flows are feeding the MDCs with pre-shock material originating from the diffuse medium, which contains $\mathrm{Si}$ in the gas phase due to the exposition to the radiation field of nearby $\mathrm{OB}$ stars, or even that in some cases there is some $\mathrm{SiO}$ released into the gas phase due to shocks from earlier high-velocity outflows. This $\mathrm{SiO}$ could then easily be maintained in the gas phase by lower velocity shocks. If this is the case, shocks with velocities below $5 \mathrm{~km} \mathrm{~s}^{-1}$ could start producing enough $\mathrm{SiO}$ to be detected. We retrieve $\mathrm{SiO}$ column densities of the order of
$0.5-1.0 \times 10^{13} \mathrm{~cm}^{-2}$ (see Sect. 3.4) for the narrow $\mathrm{SiO}$ emission (similar to what was found by Nguyên-Lu'o'ng et al. 2013), which is consistent with the possibility that $\mathrm{SiO}$ has been formed with low-velocity shocks. The low-velocity shears detected in the Cygnus-X MDCs have a line of sight velocity difference around $2-3 \mathrm{~km} \mathrm{~s}^{-1}$ (Csengeri et al. 2011a,b). Although this lies in the very lower limits to produce any $\mathrm{SiO}$ at all, these are lower limits for the actual velocity shears because projection effects are most certainly in play. Furthermore, and as mentioned in Sect. 4.2.2, since $\mathrm{SiO}$ molecules form in the post-shock gas, the actual velocities of $\mathrm{SiO}$ and the respective velocity dispersions can be different from those of the shocks, supporting the idea that the low-velocity converging flows in the region (Csengeri et al. 2011a,b) could be responsible for the widespread SiO narrow emission, despite the different observed velocities.

We therefore propose an evolutionary picture for the existence of $\mathrm{SiO}$ at systemic velocities and with a narrow line profile, not associated with outflows. In this scenario, the least centrally condensed MDCs are interpreted as being in an earlier stage of the collapse and the $\mathrm{SiO}$ would be tracing shocks from the global collapse of cloud onto the MDC. This would be the case for CygX-N40, which is a MDC with only a couple of low-mass protostars $\left(<2 M_{\odot}\right)$ at its centre, a very weak outflow, and where $90 \%$ of the $\mathrm{SiO}$ emission arises from the outskirts. This could also be the case of, for instance, IRDC G028.2300.19 (Sanhueza et al. 2013), but higher resolution observations would be necessary to confirm this. As the MDC collapses, the $\mathrm{SiO}$ emission becomes more confined to the close surroundings of denser parts, tracing the post-shock material from the shocks of the infalling MDC directly against the dense central cores. This is in essence the equivalent of the shocks from the smallscale low-velocity converging flows seen in CygX-N3, N12, and N48. At later stages, the MDC infall is less prominent and a more significant fraction of the MDC mass is already gathered in the central massive protostars. At this stage, the $\mathrm{SiO}$ luminosity is largely dominated by the powerful outflows, and there is only a weak narrow component which would point to the narrow $\mathrm{SiO}$ emission arising from the shocks from the very last remnants of the collapse of the MDC against the protostellar envelopes - this could be the case of CygX-N53 and N63. In this scenario, we would not expect to see a great amount of extended narrow $\mathrm{SiO}$ emission at systemic velocities in low-mass star-forming regions (apart from the narrow $\mathrm{SiO}$ emission intrinsically originating from high-velocity shocks), because the simple gravitational collapse of the clumps is unlikely to create shocks with enough speed to produce $\mathrm{SiO}$ (infall velocities being typically $\sim 1 \mathrm{~km} \mathrm{~s}^{-1}$ ), and these regions are generally less dynamic and less dense environments, less prone to shocks in the ISM capable of forming $\mathrm{SiO}$. This is consistent with the fact that the $\mathrm{SiO}$ emission detected so far in low-mass star-forming regions is associated with high-velocity protostellar outflows, either as broad $\mathrm{SiO}$ emission, or as narrow emission with a velocity peak offset from systemic velocities (e.g. Gueth et al. 1998; Nisini et al. 2007; Gusdorf et al. 2011; Gómez-Ruiz et al. 2013).

Even though this dynamical view would predict a smaller amount of widespread $\mathrm{SiO}$ narrow emission at systemic velocities in low-mass star-forming regions, it is sill puzzling to understand why we do not seem to observe any. If low-velocity shocks are able to form $\mathrm{SiO}$ (whether from outflows or other turbulent shocks in the ISM), then they should also be present in the low-mass case. One could think of two ways to explain the lack of detection of this component: either the composition of the gas is different in massive star-forming regions (e.g. with a greater amount of $\mathrm{Si}$ on grain mantles or in the gas phase), 
making it easier to form $\mathrm{SiO}$ with low-velocity shocks, or there is a density threshold where the lower velocity shocks become efficient at forming $\mathrm{SiO}$ only if the volume densities are sufficiently high. Hence it would be interesting to test the minimum densities needed to be able to form $\mathrm{SiO}$ through low-velocity shocks. This will be addressed in upcoming papers from Gusdorf et al. and Louvet et al. (in prep.). Observationally, progress can be made by observing higher energy transitions of $\mathrm{SiO}$ to help constrain all the characteristics of the shocks (velocities, magnetic field, pre-shock densities, chemistry, UV field, etc.).

Acknowledgements. A.D.C. acknowledges funding from the European Research Council for the FP7 ERC starting grant project LOCALSTAR. Part of the work by A.D.C. was accomplished under funding by the project PROBeS, and N.S. is supported by the project STARFICH, both funded by the French National Research Agency (ANR). T.C. acknowledges financial support for the ERC Advanced Grant GLOSTAR under contract No. 247078. IRAM is supported by INSU/CNRS (France), MPG (Germany), and IGN (Spain). The data reduction and analysis made use of the GILDAS software (http://www.iram.fr/ IRAMFR/GILDAS).

\section{References}

Anderl, S., Guillet, V., Pineau des Forêts, G., \& Flower, D. R. 2013, A\&A, 556, A69

Beuther, H., Schilke, P., Gueth, F., et al. 2002, A\&A, 387, 931

Bontemps, S., Andre, P., Terebey, S., \& Cabrit, S. 1996, A\&A, 311, 858

Bontemps, S., Motte, F., Csengeri, T., \& Schneider, N. 2010, A\&A, 524, A18

Caselli, P., Hartquist, T. W., \& Havnes, O. 1997, A\&A, 322, 296

Codella, C., Bachiller, R., \& Reipurth, B. 1999, A\&A, 343, 585

Csengeri, T., Bontemps, S., Schneider, N., Motte, F., \& Dib, S. 2011a, A\&A, 527, A135

Csengeri, T., Bontemps, S., Schneider, N., et al. 2011b, ApJ, 740, L5

Duarte-Cabral, A., Bontemps, S., Motte, F., et al. 2013, A\&A, 558, A125

Godard, B., \& Cernicharo, J. 2013, A\&A, 550, A8

Gómez-Ruiz, A. I., Hirano, N., Leurini, S., \& Liu, S.-Y. 2013, A\&A, 558, A94

Gueth, F., Guilloteau, S., \& Bachiller, R. 1998, A\&A, 333, 287

Guillet, V., Pineau Des Forêts, G., \& Jones, A. P. 2007, A\&A, 476, 263

Guillet, V., Jones, A. P., \& Pineau Des Forêts, G. 2009, A\&A, 497, 145

Guillet, V., Pineau Des Forêts, G., \& Jones, A. P. 2011, A\&A, 527, A123

Gusdorf, A., Cabrit, S., Flower, D. R., \& Pineau Des Forêts, G. 2008a, A\&A, 482, 809

Gusdorf, A., Pineau Des Forêts, G., Cabrit, S., \& Flower, D. R. 2008b, A\&A, 490, 695

Gusdorf, A., Giannini, T., Flower, D. R., et al. 2011, A\&A, 532, A53

Hennemann, M., Motte, F., Schneider, N., et al. 2012, A\&A, 543, L3

Jiménez-Serra, I., Martín-Pintado, J., Rodríguez-Franco, A., \& Marcelino, N. 2004, ApJ, 603, L49

Jiménez-Serra, I., Caselli, P., Martín-Pintado, J., \& Hartquist, T. W. 2008, A\&A, 482,549

Jiménez-Serra, I., Caselli, P., Tan, J. C., et al. 2010, MNRAS, 406, 187

Kauffmann, J., Pillai, T., \& Zhang, Q. 2013, ApJ, 765, L35

Le Picard, S. D., Canosa, A., Pineau des Forêts, G., Rebrion-Rowe, C., \& Rowe, B. R. 2001, A\&A, 372, 1064

Lefloch, B., Castets, A., Cernicharo, J., \& Loinard, L. 1998, ApJ, 504, L109

López-Sepulcre, A., Walmsley, C. M., Cesaroni, R., et al. 2011, A\&A, 526, L2

Louvet, F., Motte, F., Hennebelle, P., et al. 2014, A\&A, in press

DOI: 10.1051/0004-6361/201423603

Marston, A. P., Reach, W. T., Noriega-Crespo, A., et al. 2004, ApJS, 154, 333

Martin-Pintado, J., Bachiller, R., \& Fuente, A. 1992, A\&A, 254, 315

Motte, F., Bontemps, S., Schilke, P., et al. 2007, A\&A, 476, 1243
Nguyên-Lu'o'ng, Q., Motte, F., Carlhoff, P., et al. 2013, ApJ, 775, 88 Nisini, B., Codella, C., Giannini, T., et al. 2007, A\&A, 462, 163 Qiu, K., Zhang, Q., Beuther, H., \& Yang, J. 2007, ApJ, 654, 361 Rygl, K. L. J., Brunthaler, A., Sanna, A., et al. 2012, A\&A, 539, A79 Sanhueza, P., Jackson, J. M., Foster, J. B., et al. 2013, ApJ, 773, 123 Schilke, P., Walmsley, C. M., Pineau des Forets, G., \& Flower, D. R. 1997, A\&A, 321,293

Schilke, P., Pineau des Forêts, G., Walmsley, C. M., \& Martín-Pintado, J. 2001, A\&A, 372, 291

Schneider, N., Csengeri, T., Bontemps, S., et al. 2010, A\&A, 520, A49

Turner, B. E. 1991, ApJ, 376, 573

Walmsley, C. M., Pineau des Forêts, G., \& Flower, D. R. 1999, A\&A, 342, 542

\section{Appendix A: Examples of the SiO spectra}
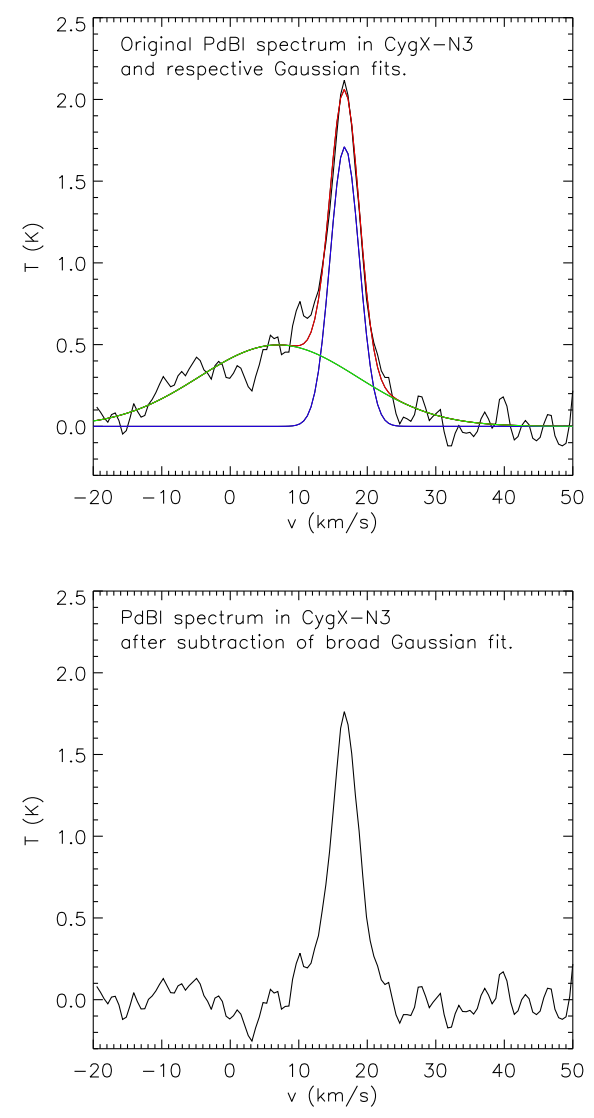

Fig. A.1. Procedure used to remove the broad high-velocity SiO emission from the datacubes. Top: example of the line fitting of a spectrum where both broad and narrow emission exist (corresponding to the blue spectrum of CygX-N3 shown in Fig. A.2). The two Gaussian fits are shown in green (for the broad component) and blue (for the narrow component). The red line shows the sum of the two Gaussian components. Bottom: same spectrum as the top panel, here with the respective broad Gaussian fit subtracted. 
A. Duarte-Cabral et al.: $\mathrm{SiO}$ emission from low- and high-velocity shocks in Cygnus-X massive dense clumps
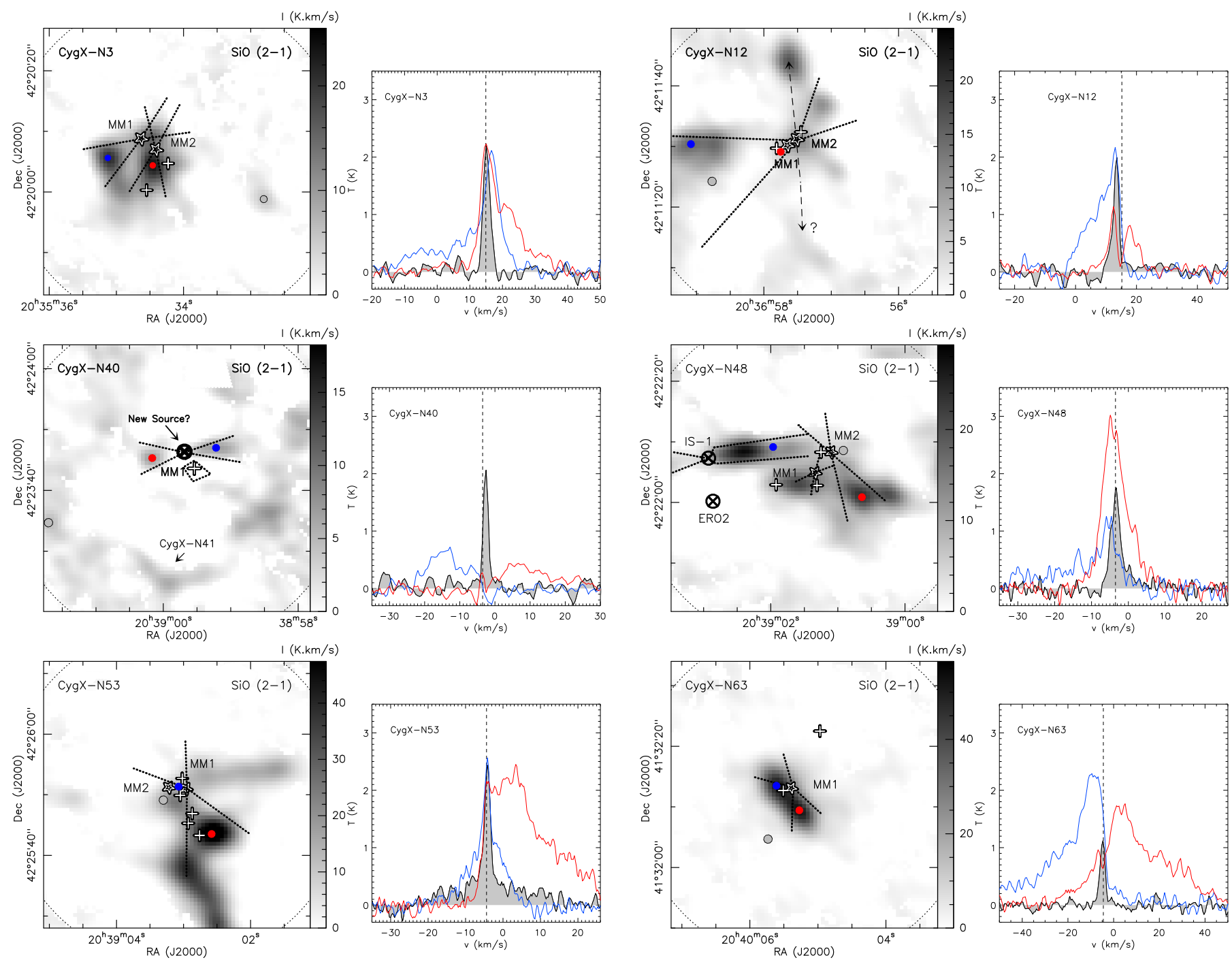

Fig. A.2. SiO emission (in units of $T_{\mathrm{mb}}$ ) of the six MDCs studied. For each MDC we show, on the left, a greyscale showing the total integrated $\mathrm{SiO}$ emission and, on the right, three overlaid spectra extracted from the datacubes. The grey, blue, and red circles in the left-hand panels show the positions of the grey, blue, and red spectra displayed on the right. The grey spectra exemplify the narrow profile of the SiO emission, and the blue and red spectra are taken at positions where there is significant wing emission. Sources and outflow directions are as labelled in Figs. 2 and 3. 